\title{
SPF: A Spatial and Functional Data Analytic Approach to cell Imaging data
}

Thao $\mathrm{Vu}^{1}$, Julia Wrobel ${ }^{1}$, Benjamin G. Bitler ${ }^{2,3}$, Erin L. Schenk ${ }^{4}$, Kimberly R. Jordan ${ }^{5}$, Debashis $\mathrm{Ghosh}^{1 *}$

1 Department of Biostatistics and Informatics, University of Colorado Anschutz Medical Campus, Aurora, CO 80045, USA

2 University of Colorado Comprehensive Cancer Center, Aurora, CO 80045, USA

3 Department of OB/GYN, Division of Reproductive Sciences, The University of Colorado, Aurora, CO 80045, USA

4 Department of Medicine, University of Colorado Anschutz Medical Campus, Aurora, CO 80045, USA 5 Department of Immunology and Microbiology, University of Colorado School of Medicine, Aurora, CO 80045, USA

* debashis.ghosh@cuanschutz.edu

\section{Abstract}

The tumor microenvironment (TME), which characterizes the tumor and its surroundings, plays a critical role in understanding cancer development and progression. Recent advances in imaging techniques enable researchers to study spatial structure of the TME at a single-cell level. Investigating spatial patterns and interactions of cell subtypes within the TME provides useful insights into how cells with different biological purposes behave, which may consequentially impact a subject's clinical outcomes. We utilize a class of well-known spatial summary statistics, the K-function and its variants, to explore inter-cell dependence as a function of distances between cells. Using techniques from functional data analysis, we introduce an approach to model the association between these summary spatial functions and subject-level outcomes, while controlling for other clinical scalar predictors such as age and disease stage. In particular, we leverage the additive functional Cox regression model (AFCM) to study the nonlinear impact of spatial interaction between tumor and stromal cells on overall survival in 
patients with non-small cell lung cancer, using multiplex immunohistochemistry (mIHC) data. The applicability of our approach is further validated using a publicly available Multiplexed Ion beam Imaging (MIBI) triple-negative breast cancer dataset.

\section{Introduction}

The tumor microenvironment (TME), which consists of tumor cells, stromal cells, immune cells and the extracellular matrix, plays a critical role in understanding cancer development and progression $[1,2]$. The concept of the TME has been around for several centuries; it was first documented by Virchow in 1863, characterizing the relationship between inflammation and tumor pathology $[3,4]$. Later, the emergence of Paget's "seed and soil" principle in 1889 further emphasized the relationship between primary tumors and their microenvironment in influencing tumor evolution [5]. However, many TME studies were not widely recognized in the field of cancer research until the late 1970s and 1980s [4]. Then, there were a number of novel discoveries indicating the influences of TME-induced signals on cancer cells and their progression [6-9] . In particular, an experimental model proposed by Tarin et al. highlighted the role of microenvironments in metastasis potential of primary tumors in mice [10]. Additionally, in 1989, Ferrara's research group discovered vascular endothelial growth factor (VEGF) and its ability to induce angiogenesis, which then became a driving force for anti-angiogenic cancer research [11].

The TME is known to be complex and heterogeneous due to the continuous cellular and molecular adaptations in primary tumor cells that allow for tumor growth and proliferation.. Accurately characterizing such heterogeneity is essential in gaining a better understanding of cancer and developing more effective treatment strategies. Conventional bulk sequencing protocols have been useful in identifying common mutation or gene expression patterns associated with cancer based on a mixture of cells from cancer samples $[12,13]$. However, such pooled approaches are not able to address the heterogeneity between distinct cell subpopulations within the TME. On the contrary, single cell profiling techniques complement the aforementioned limitations by capturing the molecular structure of the TME at a single-cell resolution, thus, provides new opportunities in translational studies $[14,15]$. As technology continues advancing, great progress has been made in high parameter imaging of tissues in situ. For example, multiplex immunohistochemistry (mIHC) detects and visualizes specific antigens in cells of a tissue section by utilizing antibody-antigen reactions coupled to a flourescent dye or an enzyme $[16,17]$. Thus, imaging allows simultaneous quantification and visualization of individual cells in tissue sections. In other words, imaging provides an additional dimension of spatial resolution to the 
single cell signature profiles.

As individual cells within the TME are genetically and epigenetically varied, they are competing with each other for space and resources (i.e., oxygen, nutrients, etc.) [18]. This is analogous to diverse species in their natural habitats as seen in ecology [19]. Typical ecological studies often involves examining spatial structures of species in a given habitat. Thus, leveraging analysis tools developed in ecology may be beneficial in studying spatial cell-cell interactions in the tumor ecosystem. For instance, Alfarouk et al. [20] highlighted regional variations in the distribution of cancer cells in relative proximity to blood vessels. This has an immediate analogy to vegetation around waterways in a riparian ecosystem. Statistics characterizing the distribution of spatial distances are typically used to investigate spatial patterns of cells in the TME [21]. For example, some quantitative measures exist, such as the Morisita-Horn index [22], which has been employed by Maley et al. [23] to capture similarity between two local communities, i.e., tumor and immune cells in breast cancer. In addition, the intratumor lymphocyte ratio is another measure used to quantify the degree of infiltration of immune cells into the tumor [24], which has been shown to have prognostic potential.

In spatial statistics terminology, a collection of cells in the TME can be considered as a point pattern generated from a point process. If the cells are a realization of a spatial process assuming complete spatial randomness (CSR), then cells do not have preference for any spatial location. In other words, cells are randomly scattered in a given region of study, in this case the TME. Under this assumption, any deviations in spatial patterns from the null model of CSR could potentially provide some useful insights into how genetically heterogeneous cells behave. In addition to spatial locations, each point in a pattern can be associated with attributes as referred to as a "mark", which can be numerical (e.g., expression intensity for a given protein, cell size, etc.) and/or categorical (e.g., cell types: immune cells, tumor cells, macrophages, etc.). Such a point pattern is known as a marked point process. The K-function, a popular summary statistic proposed by Ripley [21], has been used to capture interpoint dependence with regard to distances between points in a point pattern. Several transformations of the K-function have also been introduced to explore not only spatial association of points but also the variation in the corresponding mark values. Patrick et al. recently utilizes the K-function as an exploratory analysis tool to identify and summarize complex spatial localization of multiple cell types within the TME, with applications on multiplexed imaging cytometry data [25]. Similarly, Canete et al. introduced the R package spicyR to relate changes in spatial localization of different cell types across subjects with disease progression [26], utilizing a localization score. The score, which is calculated as a integrated difference between the observed and expected L-function, i.e., the variance stabilized K-function, summarizes the spatial 
attraction or avoidance between pairs of different cell types. Following a different approach, Barua et al. $\quad 60$ considers the G-cross function to quantitatively differentiate the colocalization between tumor cells and ${ }_{61}$ infiltrative immune cells versus tumor cells and noninfiltrative cells as a function of cell distance [27]. 62 Area under G-cross curves (AUC) is then incorporated with clinical factors including patient age, smoking history, and disease stage to find association with patient overall survival through univariate Cox proportional hazard regression model [28]. However, summarizing each curve into a single value might lose some information regarding the progression patterns as the inter-cell distance increases.

Herein, we introduce an approach, which leverages both spatial statistic summaries as well as a recently published model, the Additive Functional Cox Model (AFCM) [29], to incorporate spatial heterogeneity of cells available in histological images as functional covariates in a regression framework. Such an approach allows for the addition of other clinical variables to study their impact on patient survival. More precisely, we employ a derivative of the K-function, the mark connection function (mcf) [30], to qualitatively investigate the spatial patterns of the "seed" and "soil" factors in the TME such as primary tumor versus stromal cells, primary tumor versus immune cells, etc. In addition, the correlation in the expression level of each marker between neighboring cell types can be quantified using Moran's I statistic [31]. Depending on the research problem, the proposed framework provides the flexibility in modelling qualitative and/or quantitative spatial information. The remainder of the article is organized as follows. Section 2 describes in detail summary spatial functions and how to incorporate them into the Cox model. Section 3 presents the results on real datasets, and Section 4 describes the simulation framework. Finally, we provide concluding remarks and discussion in Section 5.

\section{Methods}

\subsection{Spatial summary functions}

\section{Summary functions for qualitatively marked point patterns}

Qualitatively marked patterns consist of different types of points. An example includes cells in a typically employed to summarize any important feature of a given point pattern such as nearest-neighbor distance, which measures the average distance from a cell of one type to its closest cell of other type in the same pattern. Furthermore, some spatial functions are also used to further detect and quantify patterns using the density of points that are $r$ units apart. In particular, the mark connection function 
(mcf) $[30,32]$ is used to reveal interesting relationships between points belonging to two different types $i$ and $j$, referred to as cross-type points.

Let $g_{i j}(r)$ be the pair correlation function between types $i$ and $j$ and $g(r)$ be the pair correlation function for an unmarked process which disregards any label associated with points in the pattern. This pair correlation function $g(r)$ is used as an alternative to the K-function and describes the distribution of interpoint distances equal to $r$ and, for the unmarked process, is defined by

$$
g(r)=K^{\prime}(r) / 2 \pi r
$$

where $K^{\prime}(r)$ is the derivative of the K-function with respect to distance $r$. Similarly,

$$
g_{i j}(r)=K_{i j}^{\prime}(r) / 2 \pi r
$$

with $K_{i j}^{\prime}(r)$ being the derivative of $K_{i j}(r)$. Note that $K_{i j}^{\prime}(r)$ is the cross-type K-function capturing the expected number of points of type $j$ lying within $r$ unit distance of a typical point of type $i$.

Then, we define the mcf as

$$
m c f_{i j}(r)=\frac{P(\text { point of type } i \text { in } \mathrm{U}, \text { point of type } j \text { in } \mathrm{V})}{P(\text { point in } \mathrm{U}, \text { point in } \mathrm{V})}=\frac{\lambda_{i} \lambda_{j} g_{i j}(r)}{\lambda_{\bullet} g(r)}
$$

where $\lambda_{i}$ and $\lambda_{j}$ are intensity functions of types $i$ and $j$ respectively; $\lambda_{\bullet}$ is the intensity function of unmarked point process. $\mathrm{U}$ and $\mathrm{V}$ are two separate subsets of the pattern, referred to as subpatterns, which contain points of types $i$ and $j$, respectively. Here, $\lambda_{i}$ and $\lambda_{j}$ are empirically estimated as the ratio of the number of points of $\mathrm{U}$ and $\mathrm{V}$ and the image area $\mathrm{W}$. In other words, $\lambda_{i}=n(u) /|W|$ and $\lambda_{j}=n(v) /|W|$ with $n(u)$ and $n(v)$ the number of observed points in $u$ and $v$ respectively, and $|W|$ as the area of an observed image. Similarly, the unmarked intensity is estimated as the sum of individual marked intensity functions in a point process, as $\lambda_{\bullet}=\lambda_{i}+\lambda_{j}$. Higher values of the mark-connection function denote more cooccurrences of points of types $i$ and $j$, while the reverse holds for smaller values. Also note that the mark connection makes an assumption of isotropy, i.e., the function value between two points only depends on the distance between the two points and not on the location.

Panels (a) and (b) of Fig. 1 illustrate the distributions of tumor and stromal cells in two representative subjects, while Fig. 1 (c) and (d) display the mcf curves that capture the spatial interactions of the tumor and stroma subpatterns with respect to inter-cell distance. At short distances (small $r$ values), cells of each type tend to cluster together, leading to mcf values below the complete 
randomness indicating line at 1 . However, as $r$ increases, stromal and tumor cells start mixing in (Fig. $1 \quad 108$ (a)), which results in more cross-type cell interactions. As a result, the corresponding mcf values become 109 slightly larger than 1 toward the end of the curve (Fig. 1 (c)).

Fig 1. Examples of mark connection functions (mcf). Tumor (turquoise) and stromal (red) cell distributions of two patients' TMA slides: (a) 43 and (b) 131, respectively. Corresponding mcf curves are shown in (c) and (d), respectively with dashed horizontal lines representing mcf under complete randomness.

\section{Summary functions for numerical marks of cross-type pairs of points}

While the aforementioned mcf provides a measure of spatial proximity of cross-type points, Moran's I 112 correlation [31] incorporates additional information by taking into account a continuous value associated with each subpattern. For example, if the two subpatterns of interest are tumor and stromal cells, Moran's I allows quantification of subpattern interaction plus additional incorporation of a protein

$$
I_{m}^{i j}(r)=\frac{\sum_{s \in i} \sum_{t \in j}\left(m_{s}^{i}-\overline{m^{i}}\right)\left(m_{t}^{j}-\overline{m^{j}}\right)}{\sum_{s}\left(m_{s}^{i}-\overline{m^{\text {pool }}}\right)^{2}+\sum_{t}\left(m_{t}^{j}-\overline{m^{\text {pool }}}\right)^{2}},
$$

where $m_{s}^{i}$ is the $s$ th element of the $m$ th mark in $i$ subpattern, $m_{t}^{j}$ is the $t$ th element of the $m$ th mark in ${ }_{118}$ $j$ subpattern. Note that $\overline{m^{i}}$ and $\overline{m^{i}}$ are the mean marker intensities of type $i$ and $j$ points, respectively, ${ }_{119}$ while $\overline{m^{\text {pool }}}$ is the overall mean intensity of both types. Fig. 2 overlays Moran's I curves across all subjects, which depicts correlation in MHCII marker intensity between tumor and stromal cells as a 121 function of distance $r$ for two groups of patients in the lung cancer dataset, (a) MHCII ${ }^{h i}$ and (b) ${ }_{122}$ MHCII $^{l o}$ [33]. Similar patterns can be observed across the two panels. Specifically, tumor cells and their ${ }_{123}$ immediate neighboring stromal cells are negatively correlated in MHCII expression when they are less ${ }_{124}$ than $10 \mu \mathrm{m}$ apart. As the cross-type cell distance increases, the correlation rises at different rates, and 125 eventually drops close to 0. A more detailed discussion is included in Section 3.1.

Fig 2. Moran's I correlation between tumor-stromal cells across subjects using MHCII. (a) $\mathrm{MHCII}^{\text {hi }}$ group and (b) $\mathrm{MHCII}^{l o}$ group.

\section{$2.2 \quad$ Model}

The aforementioned functions can be considered as summarized features capturing spatial interaction 128 between individual cells for each subject. In order to investigate the association between cell-level spatial 
effect and patient survival, we leverage a previously published model, the Additive Functional Cox $\mathbf{X}_{\mathbf{i}}=\left\{X_{i}(s)\right\}_{s \in \mathcal{S}}$ which is a continuous and integrable curve defined on a compact interval. We assume that $\mathbf{X}$ is observed on a grid of points. In our setting, $\mathbf{X}_{i}$ will be a spatial summary for subject $i$, such as a mark connection function or Moran's I function. Following Cui et al. [29], we jointly model the effect of cell-level spatial interactions with clinical variables on a subject's risk of mortality as follows

where $\log \lambda_{i}\left[t ; \mathbf{Z}_{i}, \mathbf{X}_{i}\right]$ is the $\log$ hazard at time $t$, given scalar covariates $\mathbf{Z}_{i}$ and functional covariates $\mathbf{X}(s)_{i}, i=1, \ldots, n$. In Eq. $(3), \log \lambda_{0}(t)$ is the $\log$ baseline hazard function, and the parameter vector $\boldsymbol{\beta} \quad{ }_{143}$ represents a multiplicative change in $\log$ hazard ratios for a one-unit increase in $\mathbf{Z}_{i}$. We also have $F$, an ${ }_{144}$ unspecified smooth function to be estimated. As described in McLean et al. [34], $F($.$) can be modelled { }_{145}$ as a tensor product of two penalized spline bases:

$$
\log \lambda_{i}\left[t ; \mathbf{Z}_{i}, \mathbf{X}_{i}\right]=\log \lambda_{0}(t)+\mathbf{Z}_{i}^{T} \boldsymbol{\beta}+\int_{\mathcal{S}} F\left\{s, X_{i}(s)\right\} d s
$$

$$
F(s, x)=\sum_{j=1}^{K_{s}} \sum_{k=1}^{K_{x}} \theta_{j, k} B_{j}(s) B_{k}(x)
$$

where $B_{j}(s)$ and $B_{k}(x)$ are splines defined on functional domain $s$ and functional covariate domain $x, \quad{ }_{147}$ respectively. $\theta_{j, k}$ for $j=1, \ldots, K_{s} ; k=1, \ldots, K_{x}$ are spline coefficients. Following the same approach as ${ }_{148}$ Cui et al. [29], we apply cubic regression splines for both domains $s$ and $x$. Combining Eq. (3) and Eq. 149 (4), the model can be rewritten as: 


$$
\begin{aligned}
\log \lambda_{i}\left[t ; \mathbf{Z}_{i}, \mathbf{X}_{i}\right] & =\log \lambda_{0}(t)+\mathbf{Z}_{i}^{T} \boldsymbol{\beta}+\int_{\mathcal{S}} F\left\{s, X_{i}(s)\right\} d s \\
& =\log \lambda_{0}(t)+\mathbf{Z}_{i}^{T} \boldsymbol{\beta}+\sum_{j=1}^{K_{s}} \sum_{k=1}^{K_{x}} \theta_{j, k} \int_{\mathcal{S}} B_{j}(s) B_{k}\left\{X_{i}(s)\right\} d s \\
& =\log \lambda_{0}(t)+\mathbf{Z}_{i}^{T} \boldsymbol{\beta}+\mathbf{V}_{i}^{T} \boldsymbol{\theta} \\
& =\log \lambda_{0}(t)+\mathbf{W}_{i}^{T} \boldsymbol{\gamma}
\end{aligned}
$$

where, $\boldsymbol{\gamma}^{T}=\left(\boldsymbol{\beta}^{T}, \boldsymbol{\theta}^{T}\right)$, with $\boldsymbol{\theta}$ is a vector of entries $\theta_{j, k} ; \mathbf{W}_{i}^{T}=\left(\mathbf{Z}_{i}^{T}, \mathbf{V}_{i}^{T}\right)$, with $\mathbf{V}_{i}^{T}$ is a vector of entries ${ }_{151}$ $\int_{\mathcal{S}} B_{j}(s) B_{k}\left\{X_{i}(s)\right\} d s, j=1,2, \ldots, K_{s}, k=1,2, \ldots, K_{x}$. The parameters $\gamma$ are estimated by maximizing $\quad{ }_{152}$ the penalized partial log-likelihood with the smoothing parameter imposed on $\boldsymbol{\beta}$. The penalized partial ${ }_{153}$ log-likelihood is defined as follows

$$
\begin{aligned}
l_{p}(\boldsymbol{\gamma} \mid \lambda) & =l(\boldsymbol{\gamma})-\lambda J(\boldsymbol{\theta}) \\
& =\sum_{i=1}^{N} \delta_{i}\left\{\mathbf{W}_{i}^{T} \boldsymbol{\gamma}-\log \sum_{Y_{j} \geq Y_{i}} \exp \left(\mathbf{W}_{i}^{T} \boldsymbol{\gamma}\right)\right\}-\lambda J(\boldsymbol{\theta})
\end{aligned}
$$

where the penalty term can be expressed as a quadratic term $\lambda J(\boldsymbol{\theta})=\frac{1}{2} \lambda \boldsymbol{\gamma}^{T} \boldsymbol{D} \boldsymbol{\gamma}$ with $\boldsymbol{D}$ is a symmetric, non-negative definite penalty matrix. For a given smoothing parameter $\lambda$, the regression coefficients are estimated as:

$$
\hat{\gamma}(\lambda)=\arg \min _{\gamma}-l_{p}(\gamma \mid \lambda)
$$

using Newton-Raphson procedure, provided the gradient vector $\mathcal{G}=\partial l / \partial \boldsymbol{\gamma}$ and Hessian matrix $\mathcal{H}=\partial l^{2} / \partial \gamma \partial \gamma^{T}$. Following Wood et al. [35], the selection of the smoothing parameter $\lambda$ is done by optimizing the log Laplace approximate marginal likelihood, which can be expressed as

$$
\mathcal{V}(\lambda)=l_{p}(\boldsymbol{\gamma})+\log \left|\boldsymbol{D}^{\lambda}\right|_{+}-\frac{1}{2} \log |\boldsymbol{H}|+\frac{M_{p}}{2} \log (2 \pi)
$$

where, $\boldsymbol{D}^{\lambda}=\lambda \boldsymbol{D}$ and $\left|\boldsymbol{D}^{\lambda}\right|_{+}$is the product of positive eigenvalues of $\boldsymbol{D}^{\lambda}$. $M_{p}$ is the number of zero eigenvalues of $\boldsymbol{D}^{\lambda}$. The process involves optimizing $\mathcal{V}$ with respect to $\log \lambda$. More details can be found in $[35]$. 


\section{Application results}

\subsection{Lung cancer dataset}

Tissue slides collected from 153 patients with non-small cell lung cancer were sequentially stained with

Fig. 3 (a) illustrates the locations of stromal and tumor cells in XY-coordinates for a representative image. Treating a collection of individual cells within an image as a marked point pattern, a summary mark connection function using Eq. (1) was calculated to characterize the spatial relationship between primary tumor cells and tumor-associated stromal cells as a function of distance between cells. Since cell distance $r$ depended on the density of observed cells in a given image, we chose the image with the maximum number of cells to compute a reference range of distances. Fig. 3 (b) shows mcf curves for all subjects as a function of the distance between cells, measured in microns $(\mu \mathrm{m})$. These mcf curves were used as functional covariates along with scalar clinical predictors such as total number of cells, disease stage, and age in the model from Eq. (3) to obtain estimated survival time for each subject.

Fig. 3 (c) showed survival outcomes of five representative subjects measured in years with red and 
immune cells linked to better subject survival [33].

Fig 3. Lung cancer dataset. (a) Representative image illustrating locations of stromal (red) and tumor cells (turquoise) (b) Mark connection functions (mcf) for the tumor - immune cell distributions for all subjects; dashed horizontal line represents mcf under complete randomness, and (c) Survival time of five representative subjects.

Johnson et al. dichotomized lung cancer samples into high and low MHCII. In particular, specimens with more than $5 \%$ of lung cancer cells positive for MHCII were grouped into MHCII ${ }^{h i}$ while the remaining samples were classified into $\mathrm{MHCII}^{l o}$ group. The authors stated that the levels of immune infiltration increased in the MHCII ${ }^{h i}$ TME, leading to a significantly improved overall survival. Motivated by this conclusion, we computed Moran's I correlation using MHCII marker expression between tumor and stromal cells in the TME according to Eq. (2). Fig. 2 (a) and (b) illustrated the estimated Moran's I correlation curves for patients in $\mathrm{MHCII}^{h i}$ and $\mathrm{MHCII}^{l o}$ groups, respectively, as a function of distance between cross-type cells. There was slightly more variability between correlation curves of the subjects in the $\mathrm{MHCII}^{h i}$ group as compared to that in $\mathrm{MHCII}^{l o}$ group. The two groups shared a common trend of negative association in MHCII expression between tumor cells and their immediate neighboring stromal cells $(r \leq 10 \mu m)$. As the neighborhood radius expanded, the correlation increased at various rates. Instead of categorizing subjects into $\mathrm{MHCII}^{h i}$ and $\mathrm{MHCII}^{l o}$ groups before fitting the Cox proportional model, we explored the direct impact of correlation of MHCII expression across tumor and stromal cells as a function of cross-type cell distance on survival outcome. Specifically, each Moran's I correlation curve served as a functional covariate in the model (5). Fig. 4 (b) depicted the estimated surface $\hat{F}$ with values decreased from red to blue, in correspondent with a decrease in hazard of mortality, holding the remaining scalar variables fixed. If stromal and tumor cells in the neighborhood radius between 25 and $75 \mu \mathrm{m}$ were positively correlated in MHCII expression, the risk of mortality increased. However, as the positive relationship in MHCII continued past $100 \mu \mathrm{m}$, the estimated hazard of mortality decreased, linking to a better survival outcome.

Fig 4. Estimated surface from AFCM using lung cancer dataset. (a) Mcf curves and (b) Moran's I correlation using MHCII expression.

\subsection{Triple-negative breast cancer (TNBC) dataset}

We extended this approach to study the tumor-immune microenvironment in a publicly available triple-negative breast cancer (TNBC) dataset [36]. TNBC biopsies were compiled into a tissue microarray (TMA) slides and stained with 36 antibodies targeting regulators of immune activation such 
as PD1, PD-L1, etc. The slides were imaged using the Multiplexed Ion Beam Imaging (MIBI) mass processed using the $\mathrm{R}$ package raster to extract pixel coordinates. Fig. S1 (a) and (b) in the Supporting Information show two representative pixel-level images for two randomly chosen patients. Since each cell consists of multiple pixels, the average pixel-level $\mathrm{x}$ - and $\mathrm{y}$-coordinates were used to represent cell locations on each image. Fig. S1 (c) and (d) in the Supporting Information illustrate cell-level images for patients 1 and 2, respectively, with dot size proportional to cell size and color-coded for each cell type stroma regions of the TME not available, we focused on capturing the distribution of tumor and immune cells across images using mcf curves in this dataset.

Keren et al. [36] classified subjects into "compartmentalized" and "mixed" using a mixing score, which was defined as a ratio of immune-tumor interactions to the total number of immune interactions. Fig. 5 (a) and (b) depict two representative images for two categories, "mixed" and "compartmentalized", respectively. Taking a different approach, we utilized the mcf between tumor and immune cell distributions within each image to capture the tumor-immune interactions as a function of cell distance, measured in microns $(\mu \mathrm{m})$. Fig. $5(\mathrm{c})$ and $(\mathrm{d})$ illustrate the progression of such interactions as the distance between cells increased. Particularly, when tumor and immune cells mixed in (e.g., Fig 5 (a)), more interactions between tumor and immune cells were observed as the neighborhood radius expanded. As a result, the corresponding mcf curves increased at a faster rate (Fig. 5 (c)) as compared to the ones associated with the compartmentalized cell distribution (Fig. 5 (d)).

Fig 5. "Mixed" vs. "Compartmentalized" cell distributions. (a) Cell-level image of patient 11 with "mixed" cell distribution, and (b) Cell-level image of patient 5 with "compartmentalized" cell distribution; Mark connection functions (mcf) for the tumor - immune cell distribution (c) "Mixed" mcfs, (d) "Compartmentalized" mcfs; Dashed horizontal line represents mcf under complete randomness.

Within the cohort of 41 TNBC patients, two patients did not have clinical information available regarding survival outcomes. Furthermore, one patient's imaging data was corrupted with a high level of noise. As a result, only mef curves of 38 patients (Fig. 6 (a)) with corresponding distance between cells 235 were used as functional covariates in the AFCM model. Due to the small sample size, we only included 236 patient age as a scalar predictor. Fig. 6 (c) shows the survival time of five representative subjects in the study measured in years with red and turquoise symbols denoting mortality and censored, respectively. 238 The estimated functional surface $\hat{F}$ with values decreased from red to blue, corresponding to a drop from 
particular, if there were more immune cells surrounding tumor cells across all distances, which resulted

Fig 6. TNBC dataset. (a) Mark connection functions (mcf) for the tumor - immune cell distributions for all subjects in TNBC dataset; Dashed horizontal line represents mcf under complete randomness,(b) Moran's I correlation between tumor and immune cells across subjects using P53 marker expression, and (c) Survival time of five representative subjects.

Pan et al. [37] investigated the prognostic role of P53 in TNBC by applying Kaplan-Meier analyses on P53 positive and negative groups of patients. In particular, a sample was defined as P53 positive if any cancer cells was positively expressed. The authors concluded that P53 positivity associated with negative prognostic significance in breast cancer patients. In a different manner, we attempted to study the impact of the relationship between tumor and immune cells in the TME with respect to the P53 ${ }^{251}$ expression on patient survival. By considering tumor and immune cells in the TME as two subpatterns, ${ }^{252}$ we calculated the Moran's I correlation using P53 marker expression as a function of between-pattern cell 253 distance according to Eq. (2). Fig. 6 (b) show the correlation in P53 expression between tumor and ${ }^{254}$ immune cells in the TME as a function of cell distance, measured in microns $(\mu \mathrm{m})$ across 38 patients. In 255 a few patients, immune and tumor cells in the TME, which were less than $100 \mu \mathrm{m}$ apart, were positively ${ }^{256}$ correlated. As cell distance became larger, the correlation dropped close to 0. Consequently, each ${ }^{257}$ Moran's I correlation curve served as functional covariates in the AFCM model in (5) in conjunction with age as a scalar predictor. The estimated functional surface $\hat{F}$ shown in Fig. 7 (b) had the values decreased from red to blue, in correspondence with a decrease in hazard of mortality, while holding the ${ }_{260}$ scalar variable fixed. Interestingly, positive correlation in P53 expression between immune and tumor 261 cells across distances up to $260 \mu \mathrm{m}$ associated with a decline in estimated risk of mortality. Additionally, ${ }^{262}$ the higher the positive association in P53 expression between immune and tumor cells, which were less than $100 \mu \mathrm{m}$ apart, the better the estimated survival.

Fig 7. Estimated surface from AFCM using TNBC dataset. (a) Mcf curves and (b) Moran's I correlation using P53 expression.

Section S.2 of the Supporting Information includes an additional analysis using a Vectra dataset of 265 114 ovarian cancer patients [38]. We followed the same approach to investigate the impact of spatial ${ }^{266}$ distribution of cells in tumor and stroma regions of the TME on patient overall survival. Furthermore, ${ }^{267}$ 
the correlation in Ki67 marker expression between tumor and stromal cells in close proximity was

\section{Simulation Study}

\subsection{Setup}

We performed simulation studies to evaluate the finite-sample properties of the proposed methodology. $\quad 272$

We considered a scenario with one functional covariate $\mathbf{X}_{\mathbf{i}}$ and one scalar covariate $Z_{i}$ for simplicity. ${ }^{273}$ Model (3) can be rewritten as:

$$
\begin{aligned}
& \log \lambda_{i}\left(t ; Z_{i}, \mathbf{X}_{i}\right)=\log \lambda_{0}(t)+Z_{i} \beta+\int_{\mathcal{S}} F\left\{s, X_{i}(s)\right\} d s \\
& \log \lambda_{i}\left(t ; Z_{i}, \mathbf{X}_{i}\right)=\log \lambda_{0}(t)+\eta_{i}
\end{aligned}
$$

Both scalar and functional terms were simulated directly from the lung cancer dataset to mimic

real-world parameter settings. More specifically, the scalar predictor $Z_{i}^{*}$ was simulated from the normal distribution with mean and standard deviation obtained empirically from the distribution of age.

Additionally, the functional covariates $X_{i}(s)$ were simulated empirically by applying FPCA [39] to the estimated mcf curves. Following Cui et al. [29], we simulated the functional covariates as $X_{i}^{*}(s)=\hat{\mu}(s)+\sum_{j=1}^{M} \sqrt{\hat{\lambda}_{j}} e_{i j} \hat{\phi}_{j}(s)$ with M being the number of principal components. In the definition of $X_{i}^{*}(s), e_{i j}$ were random Gaussian noise with mean 0 and variance one. The mean function $\hat{\mu}(s), \quad{ }^{281}$ eigenvalues $\hat{\lambda}_{j}$, and eigenfunctions $\hat{\phi}_{j}(s)$ were computed by applying FPCA on the estimated mcf curves ${ }^{282}$ using the package refund in $\mathrm{R}[40]$. By specifying the scalar coefficient $\beta=1$ and the functional form ${ }^{283}$ $F=X^{3}(s)$, the simulated linear predictor was generated as $\eta_{i}^{*}=Z_{i}^{*}+\sum_{s \in \mathcal{S}} X_{i}^{* 3}(s) s$. With the prespecified functional form $F$, we scaled the functional domain $\mathcal{S}$, which represented the distance between cells, to $[0,1]$. The functional covariate values $\mathcal{X}$ were kept in the range $[0,1.2]$. Doing so prevented the simulated linear predictor from being dominated by either term. From the fitted model in Section 3.1, we obtained the estimated cumulative baseline hazard $\int_{0}^{t} \lambda_{0}(x) d x$, which was then used to generate a survival function for each individual, such that $\tilde{S}_{i}(t)=\exp \left\{-e^{\eta_{i}^{*}} \int_{0}^{t} \lambda_{0}(x) d x\right\}$. The estimated 289 survival times $T_{i}^{*}$ were generated from the survival function; and the censoring times $C_{i}^{*}$ were simulated based on the empirical distribution of the observed censoring times. 


\subsection{Predictive Performance}

Four datasets of different sizes $(\mathrm{N}=1000,500,200$, and 100, respectively) were simulated following the procedure in Section 4.1. Each dataset was partitioned into training (75\%) and testing (25\%) sets. Three ${ }^{294}$ models were fit using the training set: (1) AFCM model with both scalar and functional terms, (2) 295 AFCM model with only functional term, and (3) regular Cox proportional hazard model with only scalar ${ }_{296}$ term. The predicted linear predictor $\hat{\eta}_{i}{ }^{(u)}, u=1,2,3$ was obtained from the testing set for the $u$ th ${ }^{297}$ model. At each sample size, mean squared errors $M S E^{(u)}$ was computed as the average of squared ${ }^{298}$ differences between the predicted $\hat{\eta}_{i}{ }^{(u)}$ and the "true" linear predictor $\eta_{i}^{*}$ such that $M S E^{(u)}=N_{t}^{-1} \sum_{i=1}^{N_{t}}\left(\hat{\eta}_{i}{ }^{(u)}-\eta_{i}^{*}\right)^{2}$, with $N_{t}$ denoting the number of subjects in the testing set.

Fig 8. Mean squared errors (MSE) across three models. Different sample sizes (a) $N=1000$, (b) $\mathrm{N}=500$, (c) $\mathrm{N}=200$, (d) $\mathrm{N}=100$.

We repeated the simulation for 100 iterations and recorded the average MSE for each of the three models across four sample sizes $\mathrm{N}=1000,500,200,100$ in Table 1. Fig. 8 displays the distribution of MSEs for the three models at each sample size, respectively. As expected, the average MSEs and corresponding standard deviations increased as the sample size decreased from 1000 to 100. More precisely, as shown in Fig. 8, the upper quartile MSE of the model (1) was less than the $25^{\text {th }}$ percentile of MSEs of the remaining two models (2) and (3), across all four sample sizes. The discrepancy between these quantiles became more apparent as the sample size increased. However, regardless of the sample size, model (1), which included both functional and scalar terms, performed substantially better than the other two models (2) and (3).

Table 1. Mean squared errors (MSE) across three different models. Different sample sizes are 1000, 500, 200, and 100, respectively. Corresponding standard deviations are recorded in parentheses.

\begin{tabular}{lllll}
\hline Model & $\mathrm{N}=1000$ & $\mathrm{~N}=500$ & $\mathrm{~N}=200$ & $\mathrm{~N}=100$ \\
\hline (1) Both & $0.64(0.49)$ & $0.9(1.09)$ & $0.73(0.80)$ & $1.00(1.13)$ \\
\hline (2) Functional covariate & $1.86(0.66)$ & $2.11(1.21)$ & $1.88(0.95)$ & $2.16(1.48)$ \\
\hline (3) Scalar covariate & $2.99(1.29)$ & $3.28(2.02)$ & $2.73(1.88)$ & $2.79(2.26)$ \\
\hline
\end{tabular}

\section{Conclusion}

Interactions between cells within the TME play a crucial role in understanding cancer development and progression. With advances in imaging technology, the additional dimension of spatial resolution is 
information between cells into a single quantitative value (e.g., Morisita-Horn index, intratumor lymphocyte ratio, etc.) before fitting a Cox proportional hazard model to quantify the association between cell profiles and survival outcome. Though easy to implement, these scalar summaries are unable to embed cell interactions as a function of spatial proximity in models that test associations with clinical outcomes. Alternatively, we consider cells with corresponding cell locations and features within the TME as marked point patterns. Then, the relative spatial organization of primary tumor cells and a variety of tumor-associated stromal cells as well as immune cells can be described through spatial summary functions such as the mark connection function (for qualitative marks) or Moran's I correlation (for quantitative marks). We propose an approach to incorporate the resulting spatial functions as functional covariates into an additive function Cox model to investigate the impact of spatial structure of cells in the TME on overall survival in addition to some clinical predictors (e.g., age, disease stage, total cell counts, etc.). We demonstrated the applicability of the proposed method by analyzing multiplex imaging datasets from three separate cancer applications collected under two different (Vectra and MIBI) 326 imaging platforms: lung cancer, ovarian cancer, and TNBC. In particular, we can flexibly use mark connection functions as a means to capture the level of stromal infiltration in the lung cancer dataset or immune infiltration in the TNBC dataset with respect to cell distance. In addition to the mcfs, continuous marker expression across cell types (e.g., tumor, immune, stromal, etc.) can be calculated using Moran's I statistics. Thus, qualitative and/or quantitative spatial summary functions can be included in the AFCM model, depending on the research question of interest. Furthermore, the advantage of integrating the spatial structure of cells in the TME and patient-level clinical information is demonstrated in the simulation study at different sample sizes.

Though we have presented promising results associating image summary spatial statistics with subjects' survival, our approach relies on the isotropic assumption, which only takes into account the relative distance between any two cells while disregarding their relative coordinations in a given image. Additionally, due to limited sample size, interactions between markers have not yet been considered in the model. Further modifications are needed to overcome such limitations. Based on locations and associated marker expression intensities of all the cells within an image, multivariate kernel density function may be used to capture the distribution of marker interactions jointly [41]. More precisely, by specifying a search radius bandwidth (e.g., 30 microns), a joint density representing marker interaction at a given location in an image is estimated using only cells within the radius such that closer cells are weighted more than distant cells. Simultaneously, the number of cells per unit area defined by the 
reflect spatially varying marker interactions across images. Due to the nature of density functions, which 346 are non-negative and integrate to one, they cannot be treated as unconstrained functional predictors as 347 in Section 2.2. Intermediate steps to transform the kernel density functions are necessary. A recent 348 approach introduced by Petersen et al. could be used to map regular density functions into a new space 349 through functional transformations including log quantile density and log hazard transformations [42]. 350 Finally, the transformed density functions are incorporated in the model as functional covariates in 351 addition to clinical predictors as before to investigate the association between spatial heterogeneity in 352 marker interactions and subject outcomes. This work is currently under investigation.

\section{Supporting Information}

S1 Fig. Representative TNBC tissue samples. Top Row: Pixel-level images of (a) Patient 1 and

S3 Fig. Ovarian cancer dataset. (a) Mcf curves for all patients, (b) Moran's I correlation between 362 tumor and stromal cells across subjects using Ki67 marker expression, and (c) Survival time of five ${ }_{363}$ representative patients.

\section{Acknowledgments}

We thank the Human Immune Monitoring Shared Resource and support of the University of Colorado 368 Human Immunology and Immunotherapy Initiative for their expert assistance in multiplex IHC and 369 generation of the ovarian and lung datasets. We acknowledge the support of the University of Colorado 370 Cancer Center Support Grant (P30CA046934). 


\section{Funding}

B.G.B. is supported by the Department of Defense Award (OC170228) and an American Cancer Society 373 Research Scholar Award (134106-RSG-19-129-01-DDC). E.L.S. is supported by NIH grant K12 374 CA086913 and ACS IRG \#16-184-56 from the American Cancer Society to the University of Colorado 375 Cancer Center, and a grant from the Cancer League of Colorado. T.V. is funded by the Grohne-Stepp 376 Endowment from the University of Colorado Cancer Center.

\section{Data and Code Availability}

The TNBC dataset is publicly available at https://www.angelolab.com/mibi-data. The mIHC

\section{Author Contributions}

Conceptualization: Thao Vu, Julia Wrobel, Debashis Ghosh.

Data Curation: Benjamin G. Bitler, Erin L. Schenk, Kimberly R. Jordan.

Formal Analysis: Thao Vu, Julia Wrobel, Debashis Ghosh.

Funding Acquisition: Benjamin G. Bitler, Erin L. Schenk, Debashis Ghosh.

Investigation: Thao Vu, Julia Wrobel, Debashis Ghosh.

Methodology: Thao Vu, Julia Wrobel, Debashis Ghosh.

Project Administration: Debashis Ghosh.

Resources: Julia Wrobel, Benjamin G. Bitler, Erin L. Schenk, Kimberly R. Jordan, Debashis Ghosh.

Software: Thao Vu.

Supervision: Julia Wrobel, Debashis Ghosh.

Validation: Thao Vu, Julia Wrobel, Debashis Ghosh. 
bioRxiv preprint doi: https://doi.org/10.1101/2021.09.29.462282; this version posted October 1,2021 . The copyright holder for this preprint (which was not certified by peer review) is the author/funder, who has granted bioRxiv a license to display the preprint in perpetuity. It is made available under aCC-BY 4.0 International license.

\section{Conflict of Interest}

None declared. 


\section{References}

1. Guo S, Deng CX. Effect of stromal cells in tumor microenvironment on metastasis initiation. International journal of biological sciences. 2018;14(14):2083.

2. Anderson NM, Simon MC. The tumor microenvironment. Current Biology. 2020;30(16):R921-R925.

3. Schmidt A, Weber O. In memoriam of Rudolf virchow: a historical retrospective including aspects of inflammation, infection and neoplasia. In: Infection and inflammation: impacts on oncogenesis. vol. 13. Karger Publishers; 2006. p. 1-15.

4. Maman S, Witz IP. A history of exploring cancer in context. Nature Reviews Cancer. 2018;18(6):359-376.

5. Paget S. The distribution of secondary growths in cancer of the breast. The Lancet. 1889;133(3421):571-573.

6. Richmond A, Thomas HG. Melanoma growth stimulatory activity: isolation from human melanoma tumors and characterization of tissue distribution. Journal of cellular biochemistry. 1988;36(2):185-198.

7. Aguirre Ghiso JA, Alonso DF, Farías EF, Gomez DE, Bal de Kier Joffè E. Deregulation of the signaling pathways controlling urokinase production: its relationship with the invasive phenotype. European journal of biochemistry. 1999;263(2):295-304.

8. Weaver V, Bissell M, Fischer A, Peterson O. The importance of the microenvironment in breast cancer progression: recapitulation of mammary tumorigenesis using a unique human mammary epithelial cell model and a three-dimensional culture assay. Biochemistry and cell biology. 1996;74(6):833-851.

9. Pohl J, Radler-Pohl A, Schirrmacher V. A model to account for the effects of oncogenes, TPA, and retinoic acid on the regulation of genes involved in metastasis. Cancer and Metastasis Reviews. 1988;7(4):347-356.

10. Tarin D, Price J. Metastatic colonization potential of primary tumour cells in mice. British journal of cancer. 1979;39(6):740-754. 
11. Leung DW, Cachianes G, Kuang WJ, Goeddel DV, Ferrara N. Vascular endothelial growth factor is a secreted angiogenic mitogen. Science. 1989;246(4935):1306-1309.

12. Zhang Y, Wang D, Peng M, Tang L, Ouyang J, Xiong F, et al. Single-cell RNA sequencing in cancer research. Journal of Experimental \& Clinical Cancer Research. 2021;40(1):1-17.

13. Fan J, Slowikowski K, Zhang F. Single-cell transcriptomics in cancer: Computational challenges and opportunities. Experimental \& Molecular Medicine. 2020;52(9):1452-1465.

14. Ren X, Kang B, Zhang Z. Understanding tumor ecosystems by single-cell sequencing: promises and limitations. Genome biology. 2018;19(1):1-14.

15. Navin N, Kendall J, Troge J, Andrews P, Rodgers L, McIndoo J, et al. Tumour evolution inferred by single-cell sequencing. Nature. 2011;472(7341):90-94.

16. Maiques O, Georgouli M, Sanz-Moreno V. Recent advances in tissue imaging for cancer research. F1000Research. 2019;8.

17. Duraiyan J, Govindarajan R, Kaliyappan K, Palanisamy M. Applications of immunohistochemistry. Journal of pharmacy \& bioallied sciences. 2012;4(Suppl 2):S307.

18. Merlo LM, Pepper JW, Reid BJ, Maley CC. Cancer as an evolutionary and ecological process. Nature reviews cancer. 2006;6(12):924-935.

19. Nawaz S, Yuan Y. Computational pathology: Exploring the spatial dimension of tumor ecology. Cancer letters. 2016;380(1):296-303.

20. Alfarouk KO, Ibrahim ME, Gatenby RA, Brown JS. Riparian ecosystems in human cancers. Evolutionary applications. 2013;6(1):46-53.

21. Ripley B. Spatial statistics: Developments 1980-3, correspondent paper. International Statistical Review/Revue Internationale de Statistique. 1984; p. 141-150.

22. Morisita M. Measuring of the dispersion of individuals and analysis of the distributional patterns. Mem Fac Sci Kyushu Univ, Ser E (Biol). 1959;2:215-235.

23. Maley CC, Koelble K, Natrajan R, Aktipis A, Yuan Y. An ecological measure of immune-cancer colocalization as a prognostic factor for breast cancer. Breast Cancer Research. 2015;17(1):1-13. 
24. Yuan Y. Modelling the spatial heterogeneity and molecular correlates of lymphocytic infiltration in triple-negative breast cancer. Journal of The Royal Society Interface. 2015;12(103):20141153.

25. Patrick E, Canete NP, Iyengar SS, Harman AN, Sutherland GT, Yang P. Spatial analysis for highly multiplexed imaging data to identify tissue microenvironments. bioRxiv.

2021;doi:10.1101/2021.08.16.456469.

26. Canete NP, Iyengar SS, Ormerod JT, Harman AN, Patrick E. spicyR: Spatial analysis of in situ cytometry data in R. bioRxiv. 2021;.

27. Barua S, Fang P, Sharma A, Fujimoto J, Wistuba I, Rao AU, et al. Spatial interaction of tumor cells and regulatory $\mathrm{T}$ cells correlates with survival in non-small cell lung cancer. Lung Cancer. $2018 ; 117: 73-79$.

28. Cox DR. Regression models and life-tables. Journal of the Royal Statistical Society: Series B (Methodological). 1972;34(2):187-202.

29. Cui E, Crainiceanu CM, Leroux A. Additive Functional Cox Model. Journal of Computational and Graphical Statistics. 2020; p. 1-14.

30. Baddeley A, Rubak E, Turner R. Spatial point patterns: methodology and applications with R. CRC press; 2015.

31. Moran PA. The interpretation of statistical maps. Journal of the Royal Statistical Society: Series B (Methodological). 1948;10(2):243-251.

32. Baddeley A, Turner R, Rubak E, Berthelsen KK. Package 'spatstat'. The Comprehensive R Archive Network (). 2014;

33. Johnson AM, Boland JM, Wrobel J, Klezcko EK, Weiser-Evans M, Hopp K, et al. Cancer cell-specific MHCII expression as a determinant of the immune infiltrate organization and function in the non-small cell lung cancer tumor microenvironment. Journal of Thoracic Oncology. 2021;

34. McLean MW, Hooker G, Staicu AM, Scheipl F, Ruppert D. Functional generalized additive models. Journal of Computational and Graphical Statistics. 2014;23(1):249-269.

35. Wood SN, Pya N, Säfken B. Smoothing parameter and model selection for general smooth models. Journal of the American Statistical Association. 2016;111(516):1548-1563. 
36. Keren L, Bosse M, Marquez D, Angoshtari R, Jain S, Varma S, et al. A structured tumor-immune microenvironment in triple negative breast cancer revealed by multiplexed ion beam imaging. Cell. 2018;174(6):1373-1387.

37. Pan Y, Yuan Y, Liu G, Wei Y. P53 and Ki-67 as prognostic markers in triple-negative breast cancer patients. PLoS One. 2017;12(2):e0172324.

38. Jordan KR, Sikora MJ, Slansky JE, Minic A, Richer JK, Moroney MR, et al. The capacity of the ovarian cancer tumor microenvironment to integrate inflammation signaling conveys a shorter disease-free interval. Clinical Cancer Research. 2020;26(23):6362-6373.

39. Silverman B, Ramsay J. Functional data analysis. In: International Encyclopedia of the Social and Behavioral Sciences. Amsterdam: Elsevier; 2001.

40. Goldsmith J, Scheipl F, Huang L, Wrobel J, Gellar J, Harezlak J, et al. refund: Regression with Functional Data. R package version 0.1-17. URL: https://CRAN R-project org/package= refund. $2019 ;.$

41. Simonoff JS. Smoothing methods in statistics. Springer Science \& Business Media; 2012.

42. Petersen A, Müller HG. Functional data analysis for density functions by transformation to a Hilbert space. The Annals of Statistics. 2016;44(1):183-218. 

bioRxiv preprint doi: https://doi.org/10.1101/2021.09.29.462282; this version posted October 1,2021 . The copyright holder for this preprint
(wh/ch was not certified by peer review) is the author/funder, who has granted bioRxiv a license to display the preprint in perpetuity. It is made which was not certified by peer review) is the author/funder, who has granted bioRxiv a license
available under $\mathrm{ACC}-\mathrm{BY} 4.0$ International license.

model

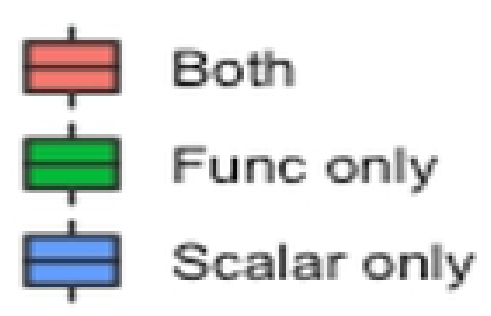

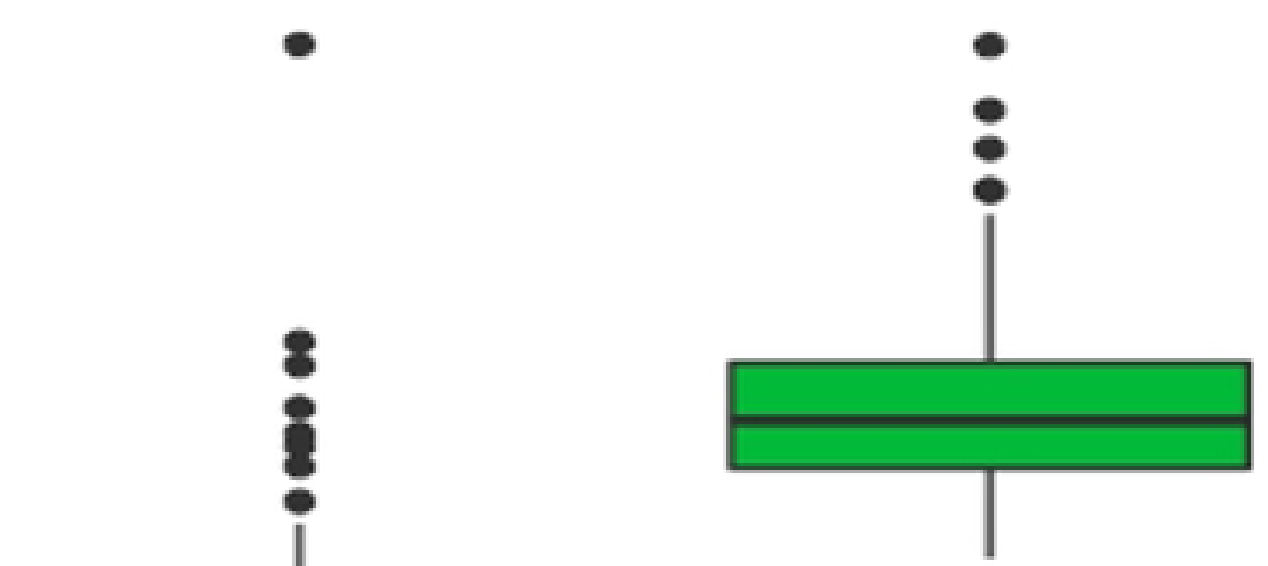

0

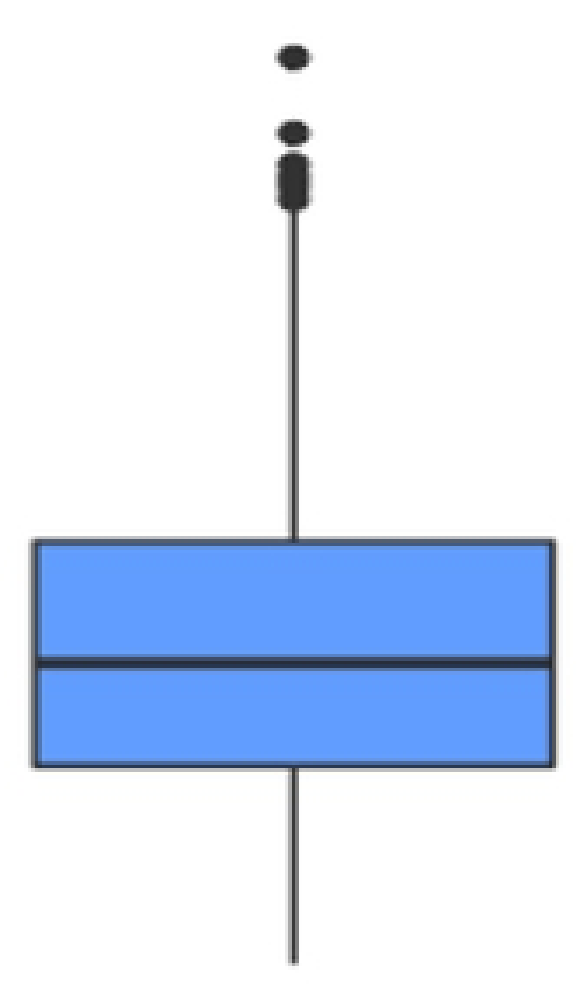

Scalar only 


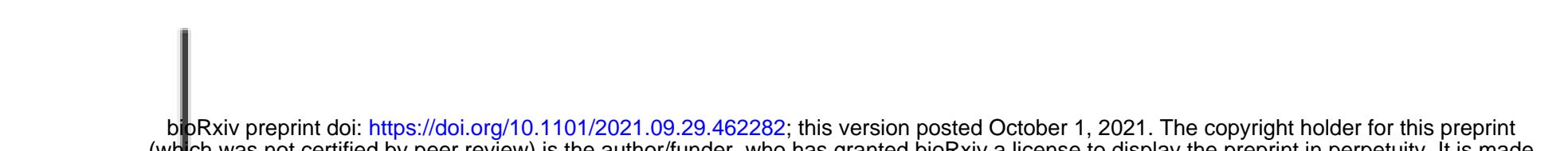

bioRxiv preprint doi: https://doi.org/10.1101/2021.09.29.462282; this version posted October 1,2021. The copyright holder for this preprint
(which was not certified by peer review) is the author/funder, who has granted bioRxiv a license to display the preprint in perpetuity. It is made

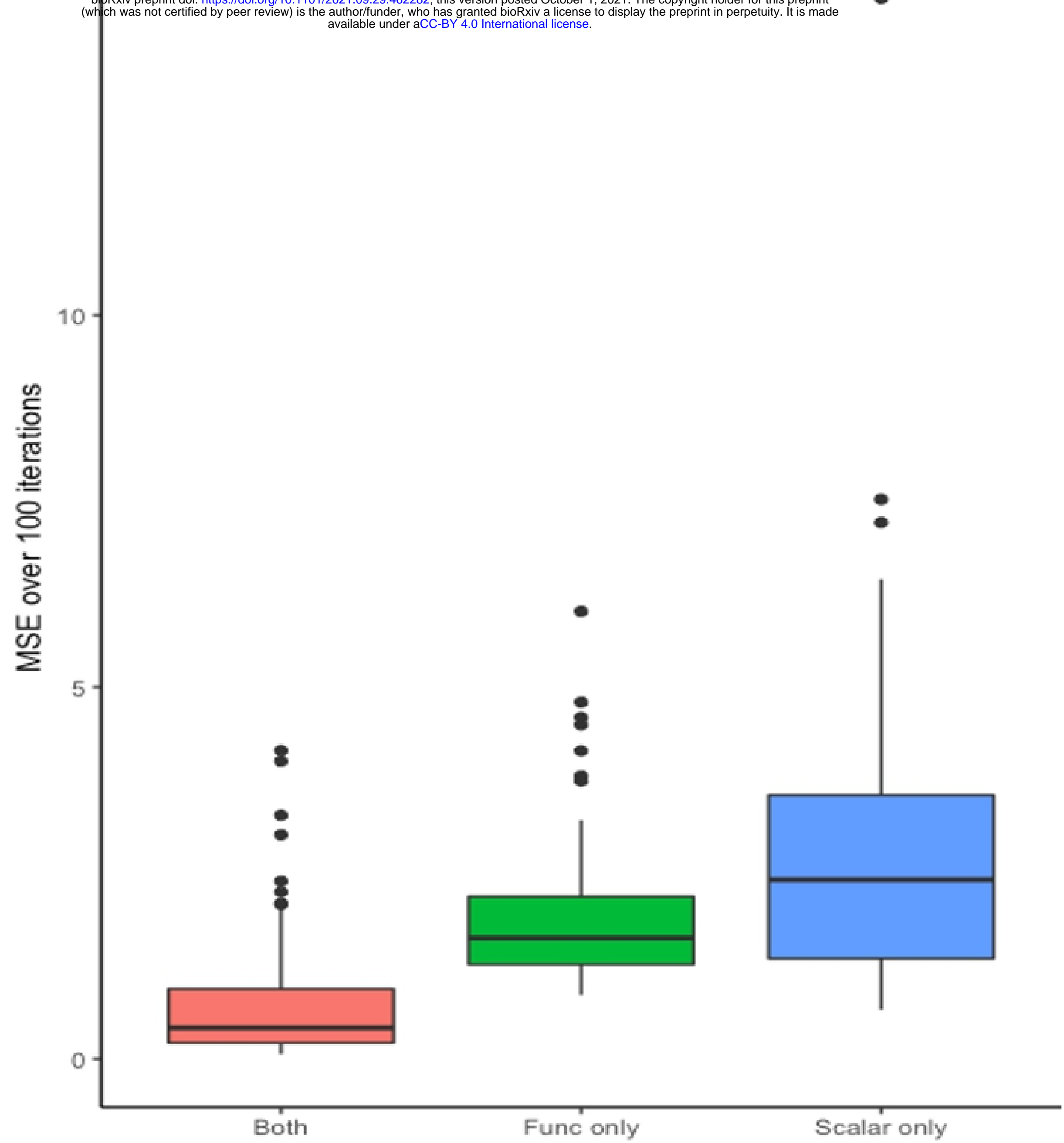

model

鸟 Both

Func only

审 Scalar only 


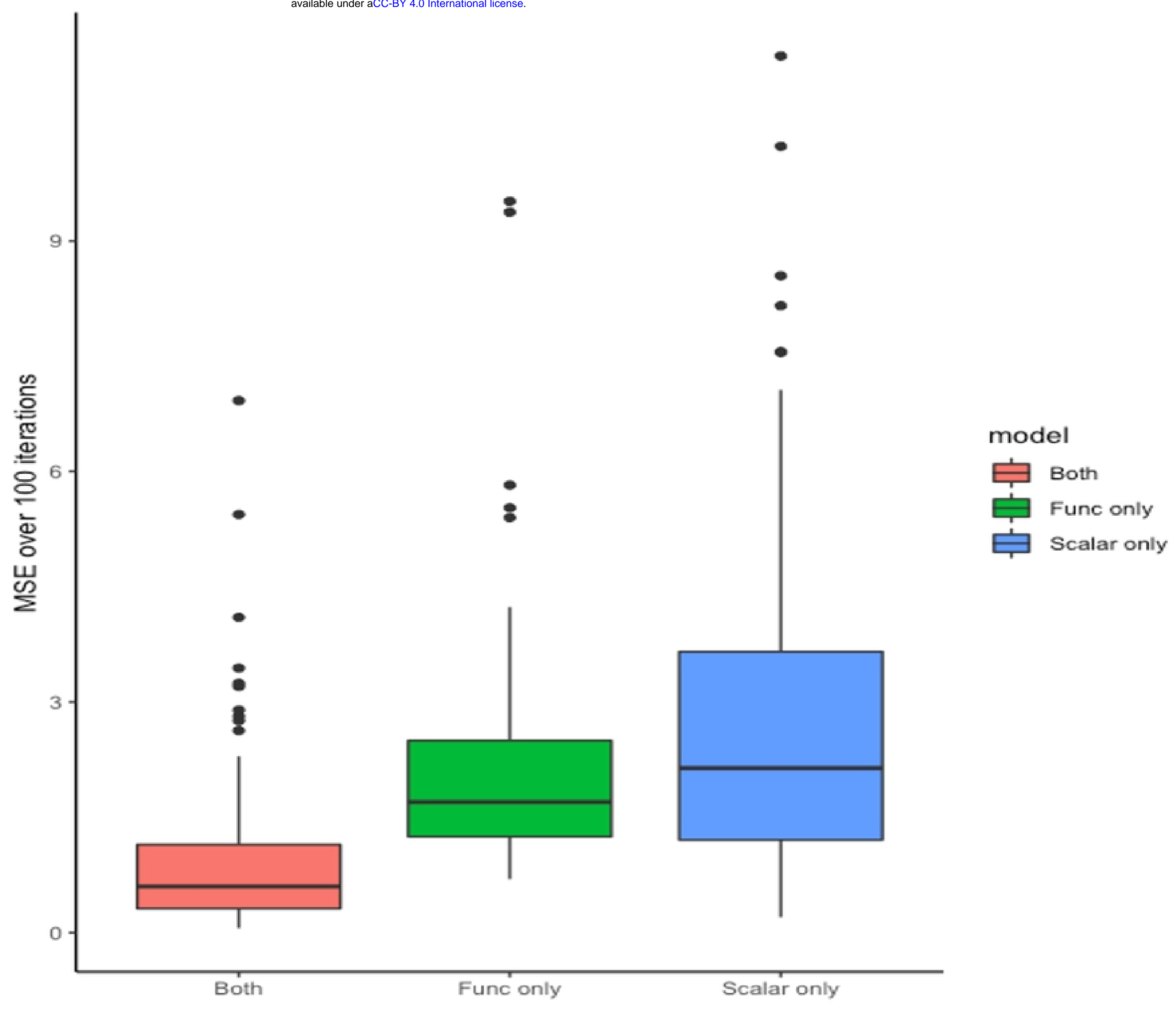




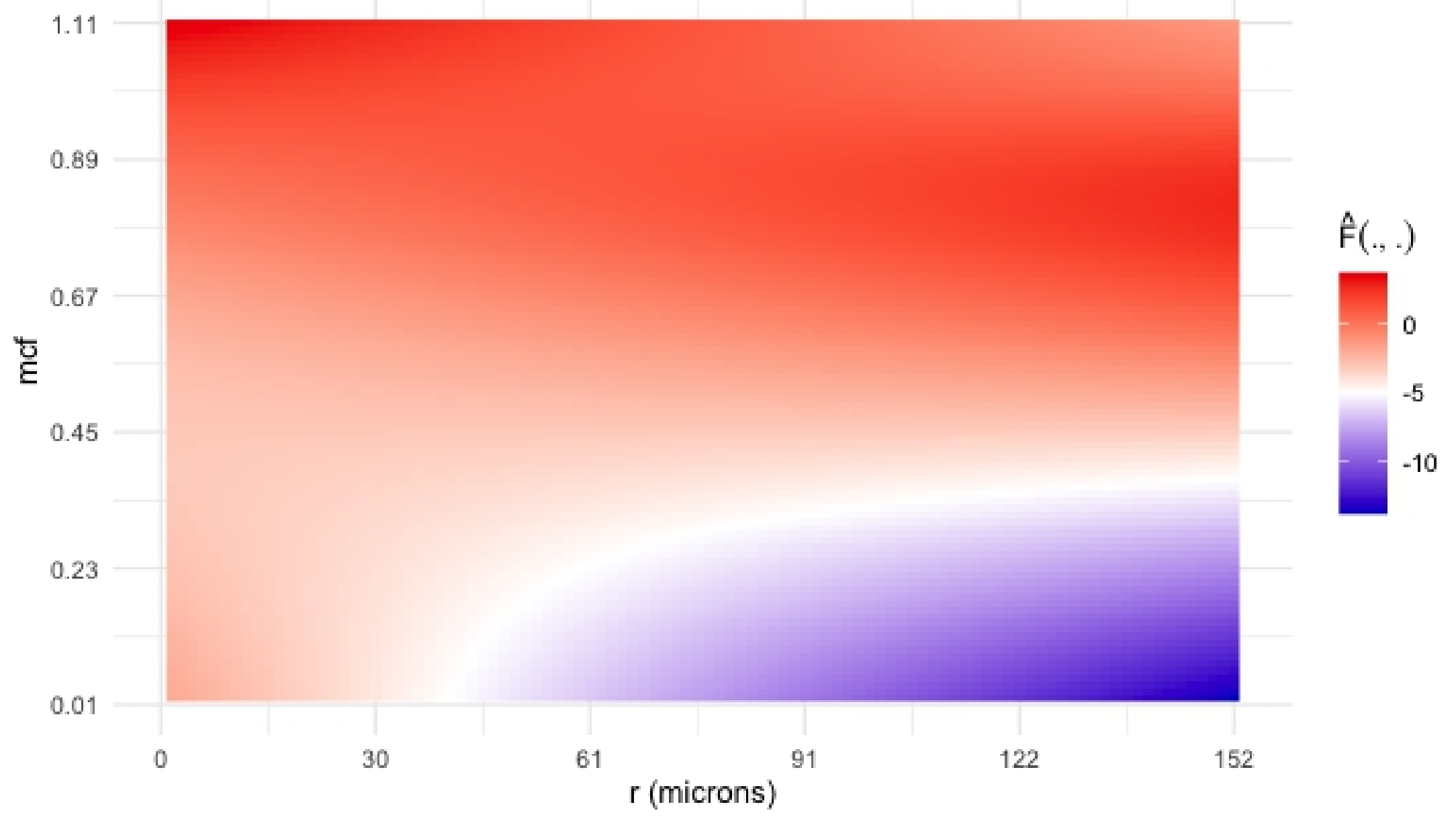





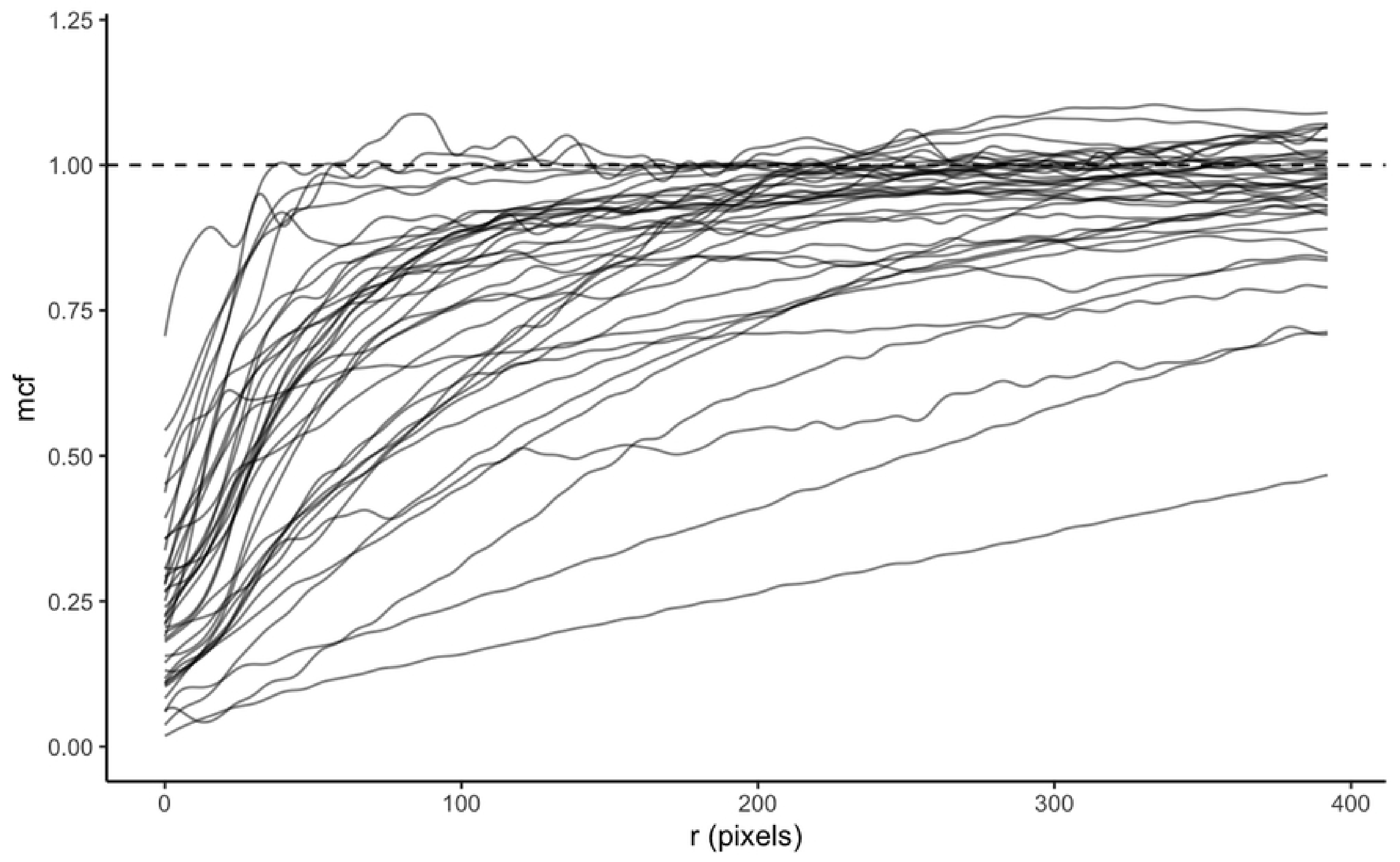




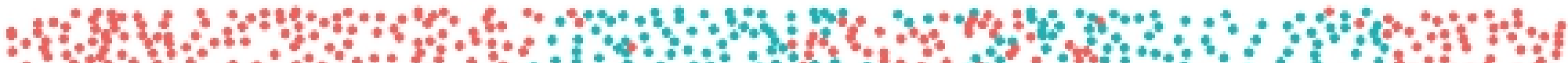
$3 x_{0}$

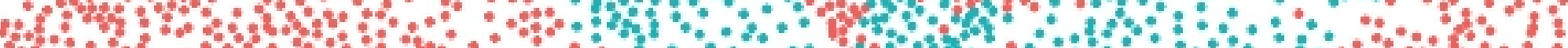

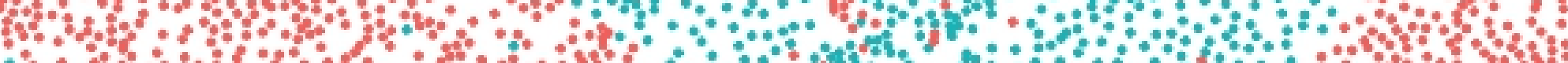
0 s. Fo Ond a

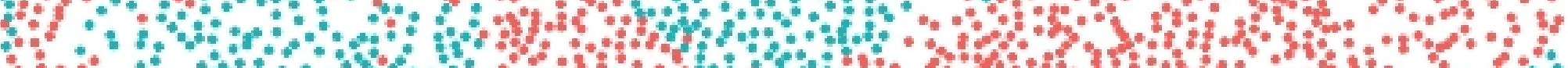
8 : $\therefore$ *

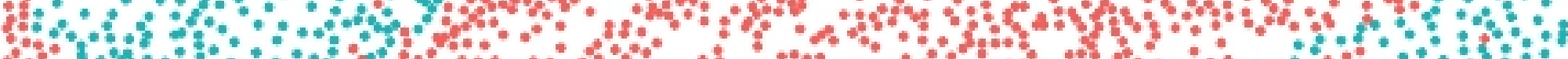

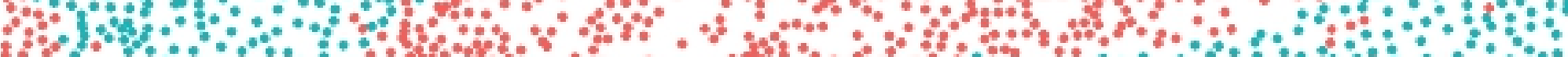
f

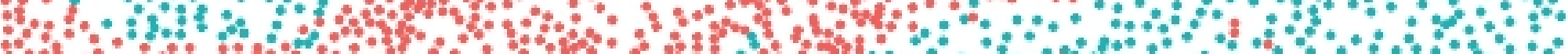
sto s.

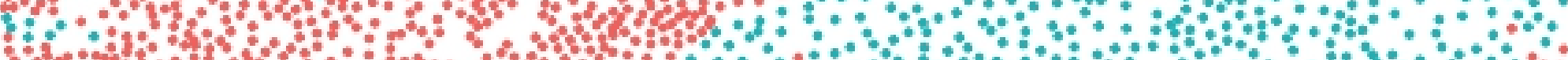

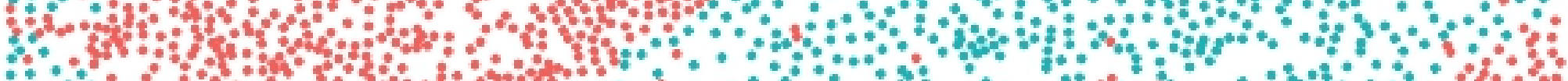

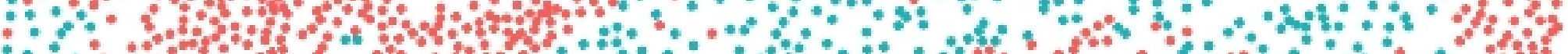
$\therefore-30^{2}$ -

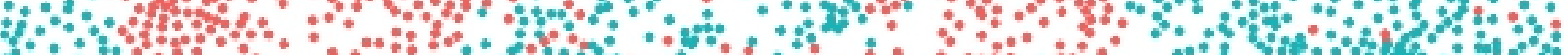
$\therefore$; 


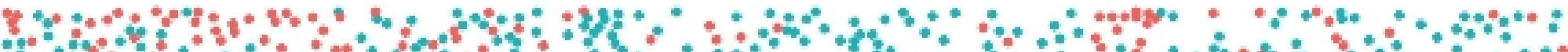
8

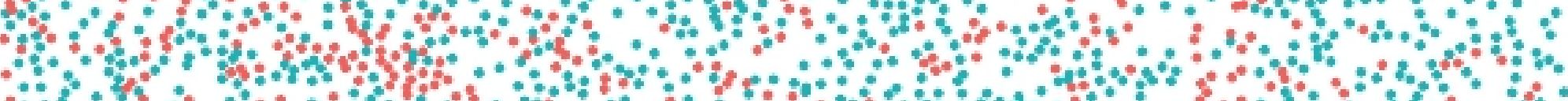

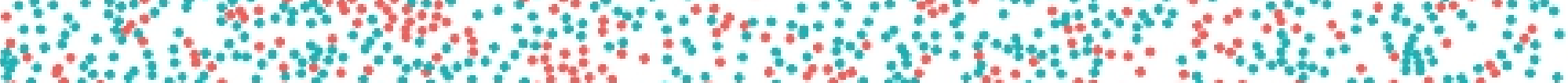
$8: 8 f^{8}$

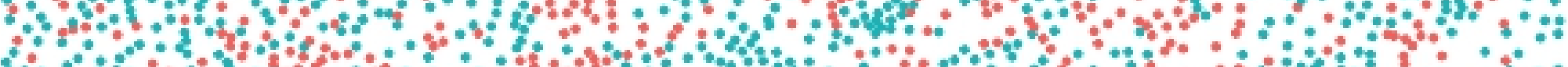
$F_{0}^{\circ}$

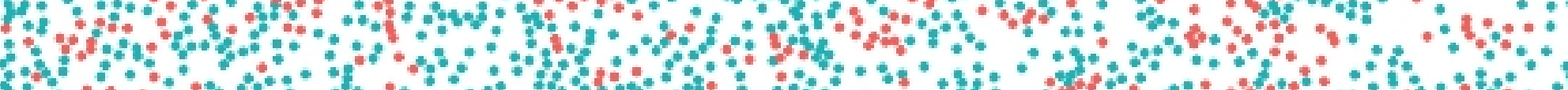

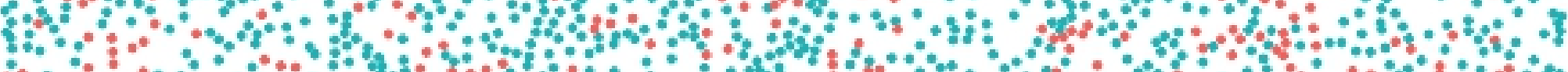

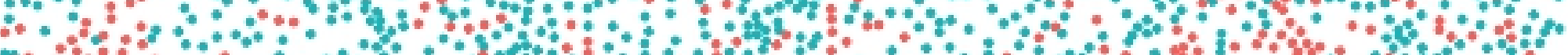
:

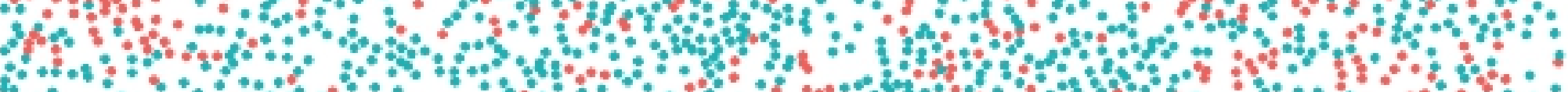

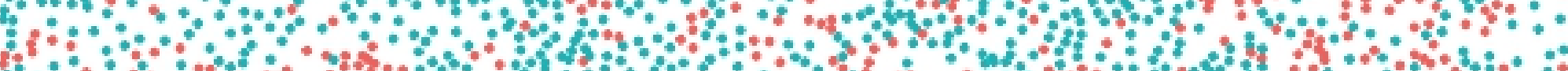

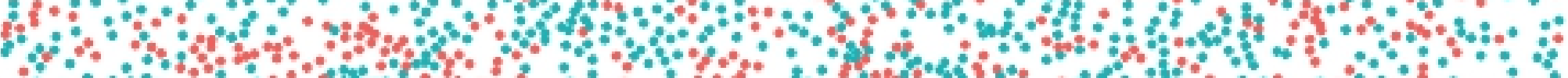

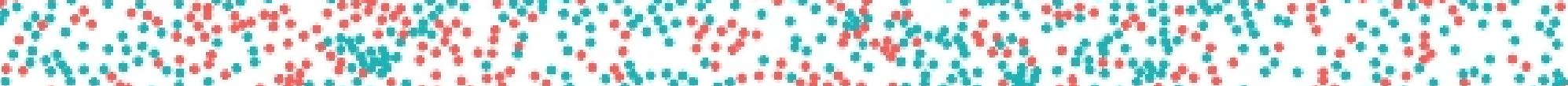

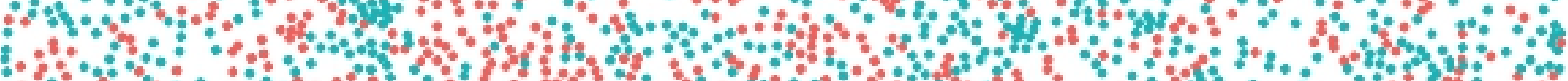
$\therefore 80^{\circ}$

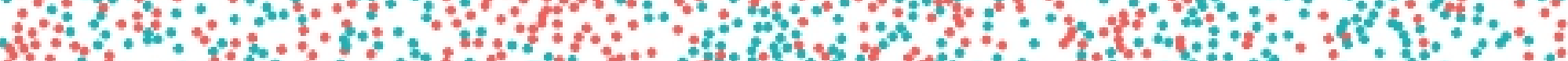

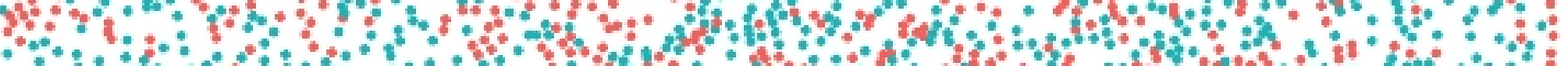

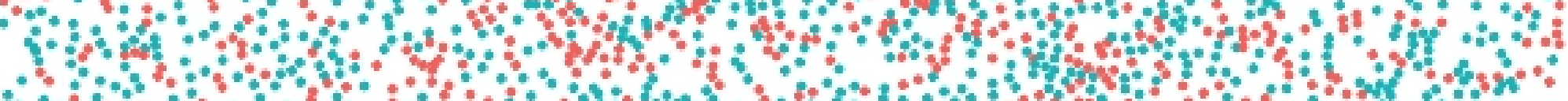
$+5$

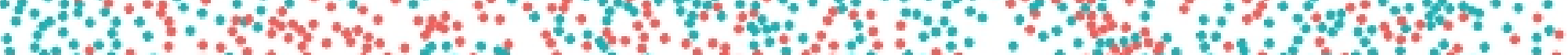

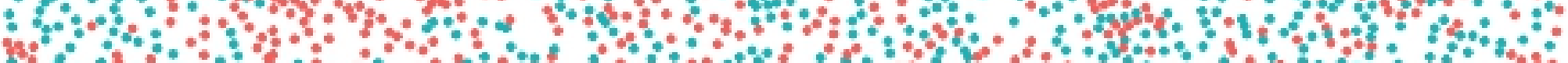

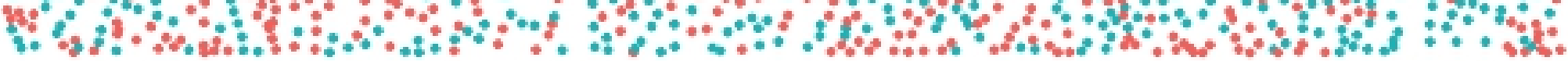




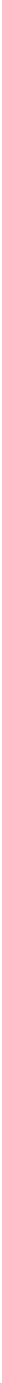




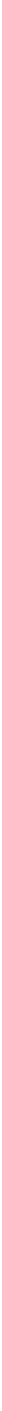




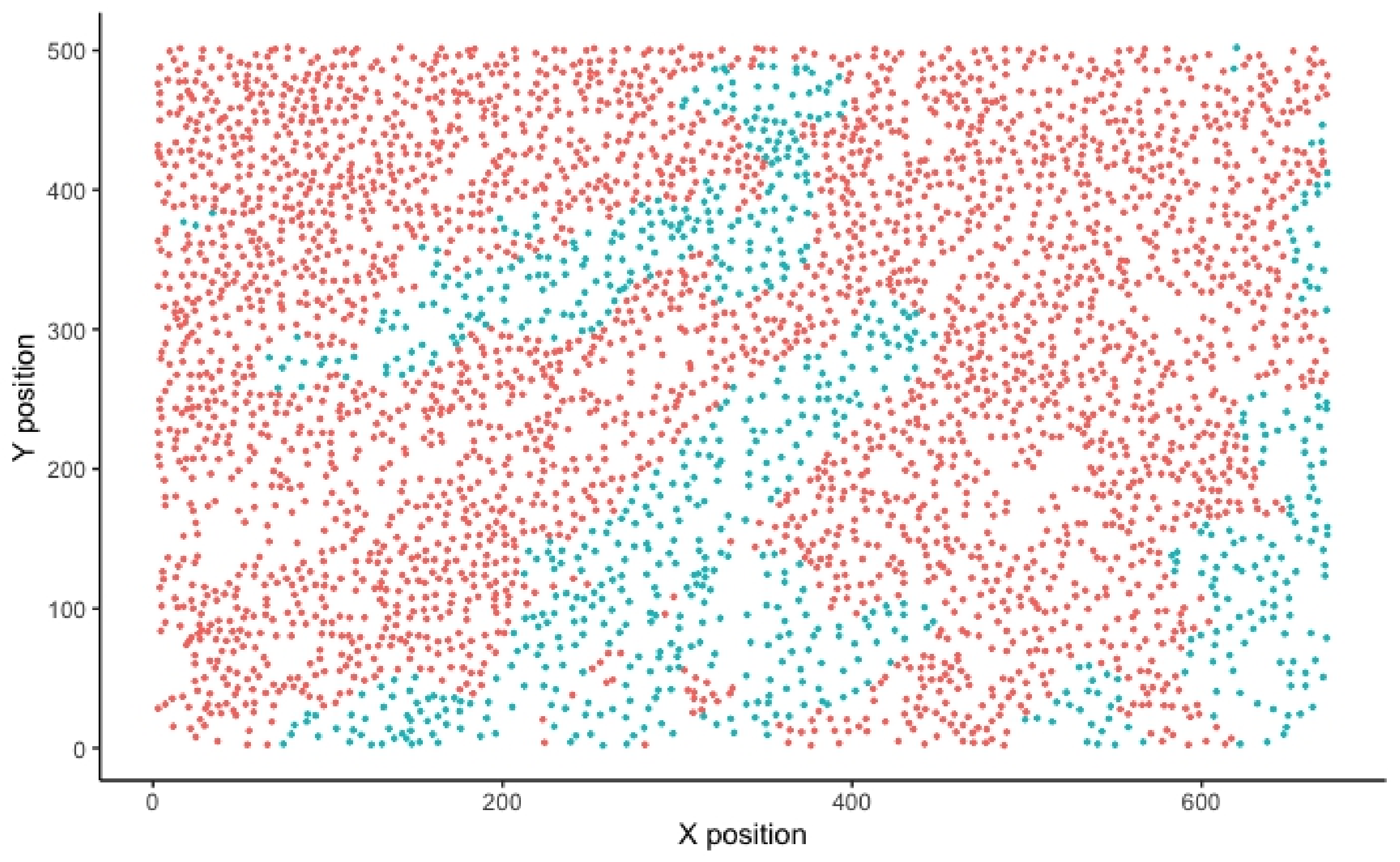




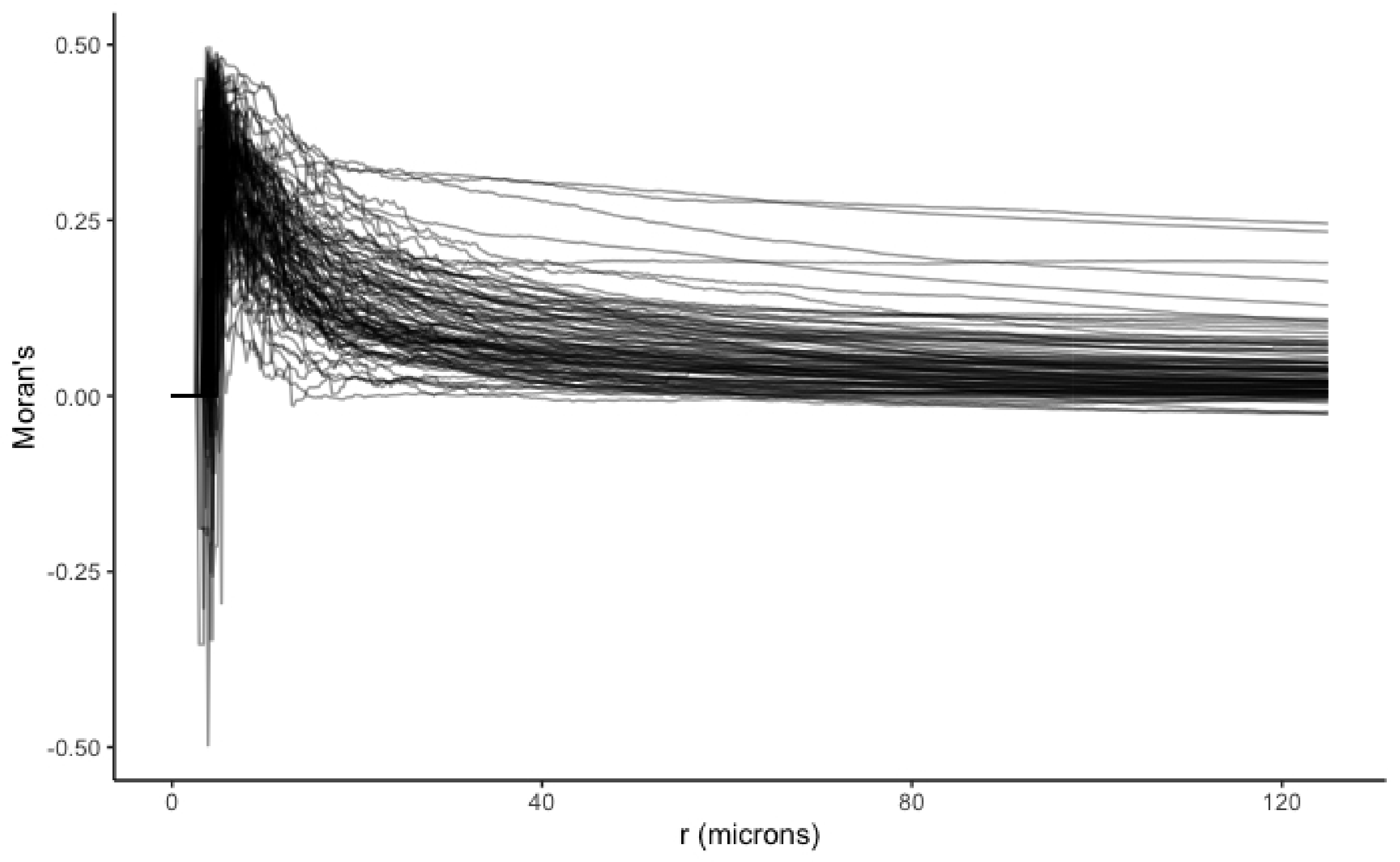




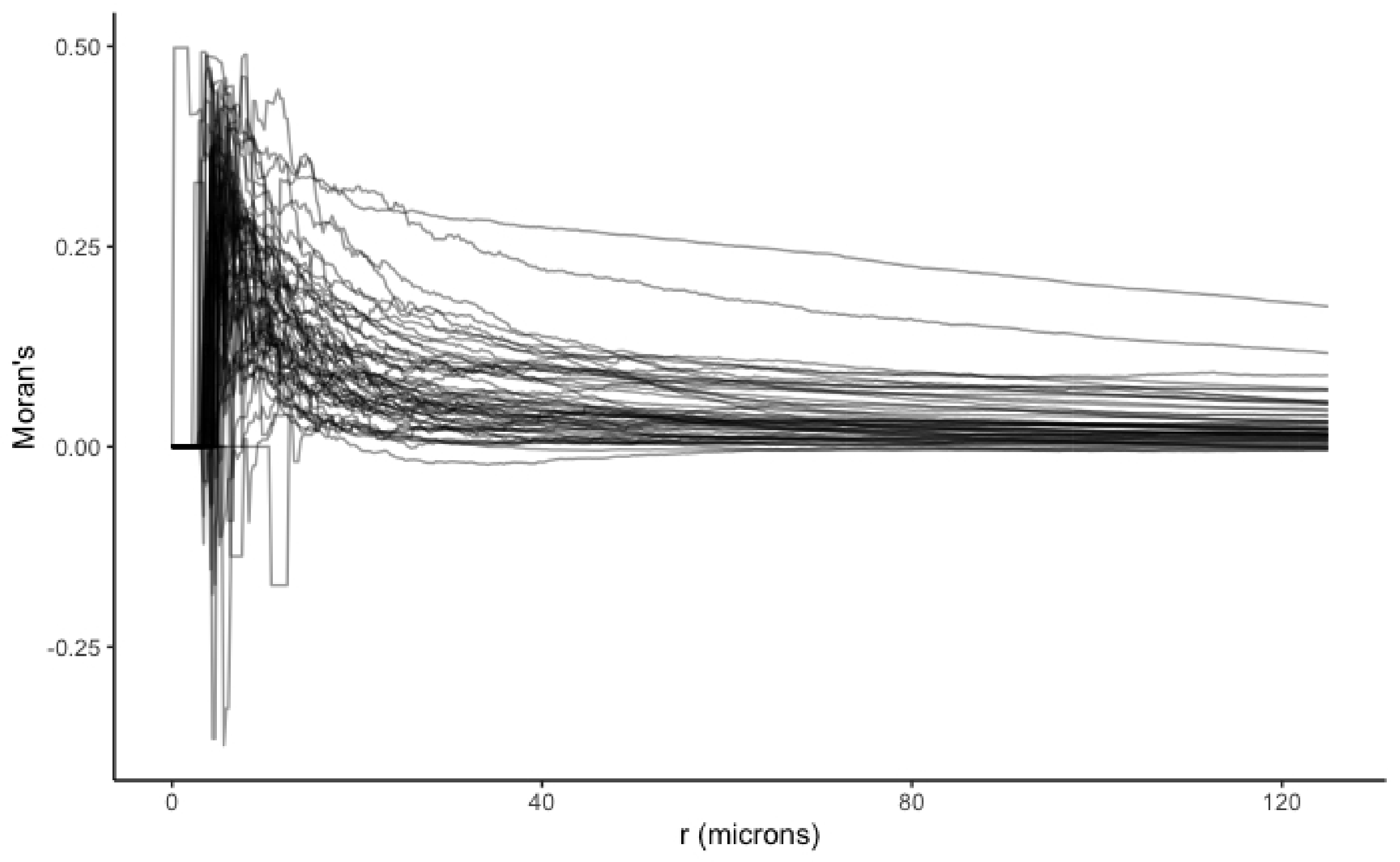




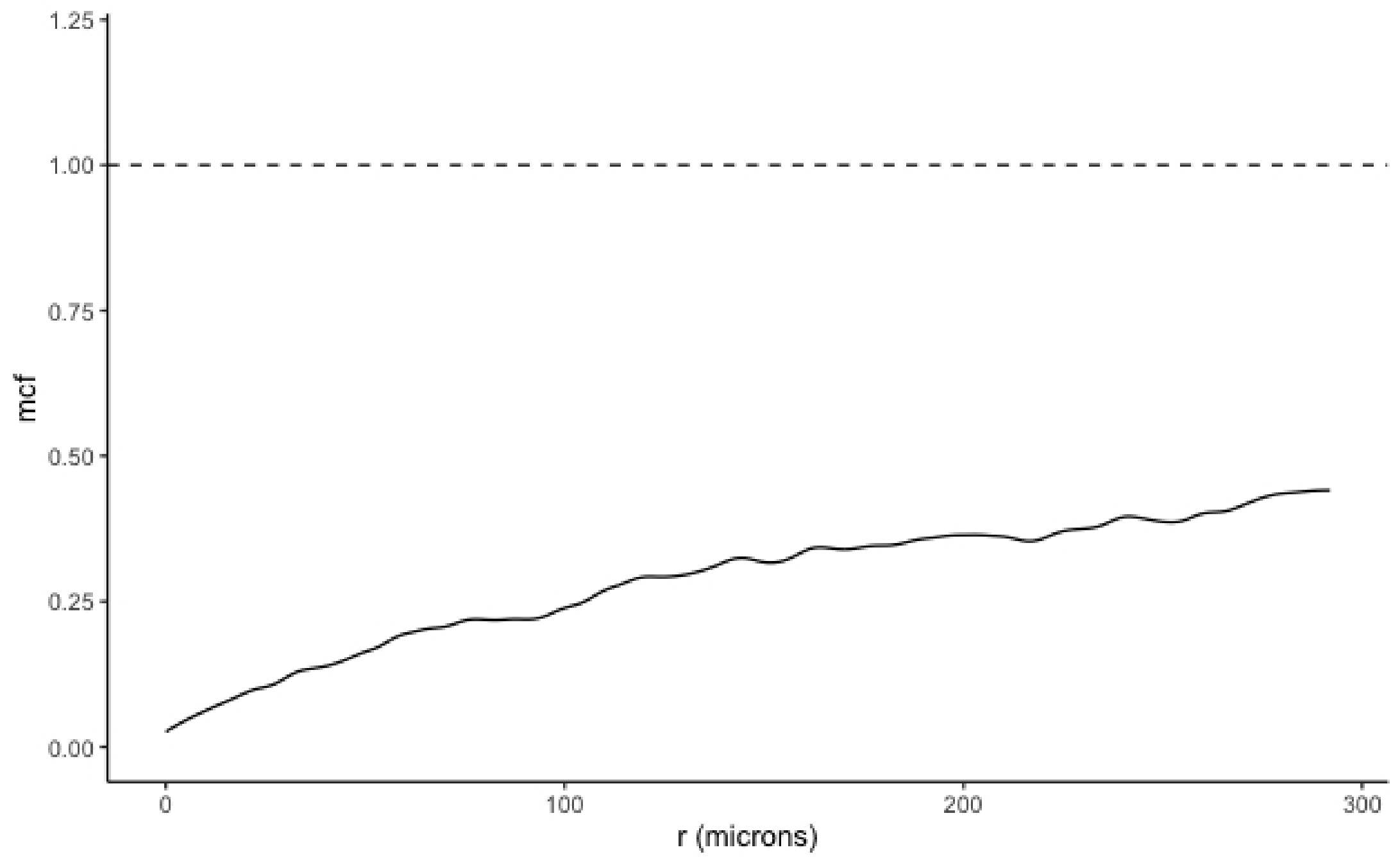




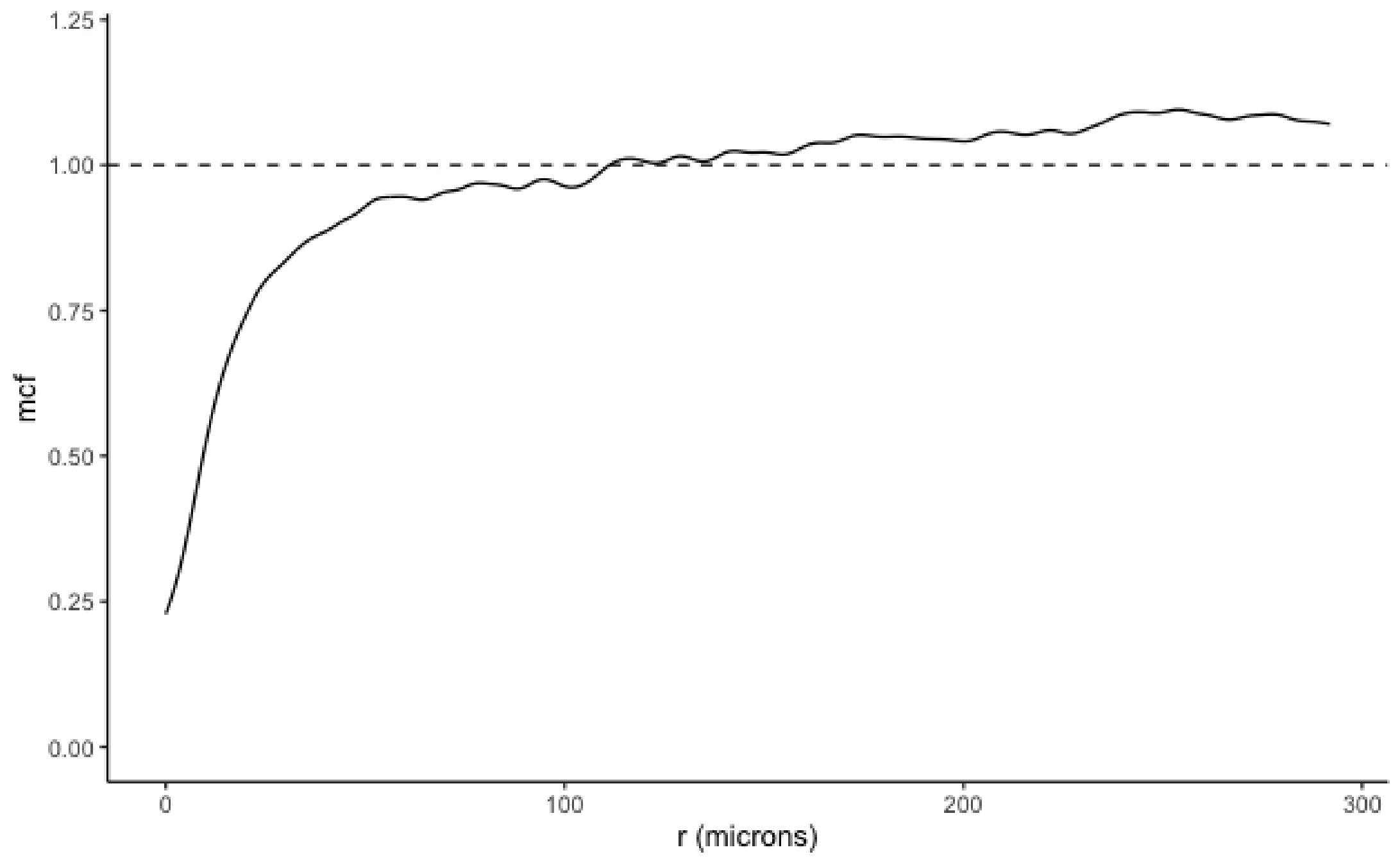


to If

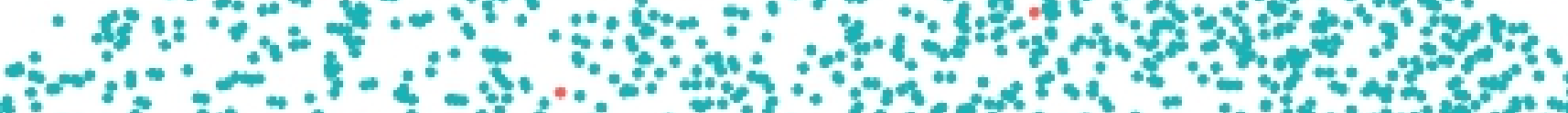

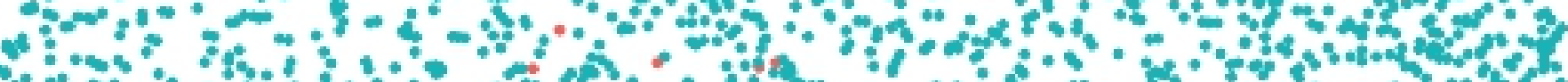

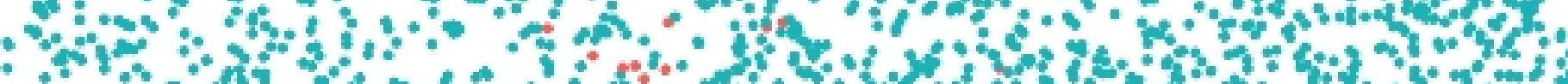
* +

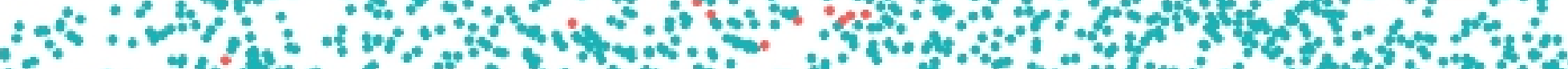

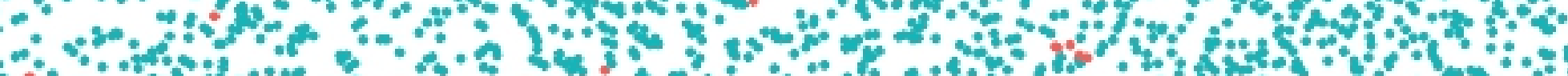
$1, \%$

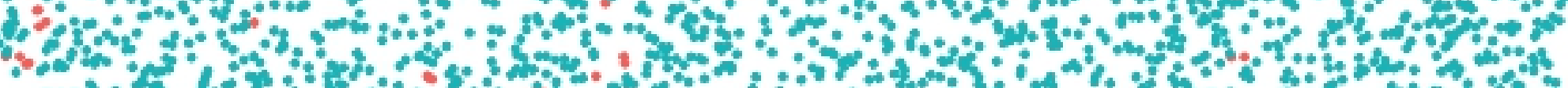

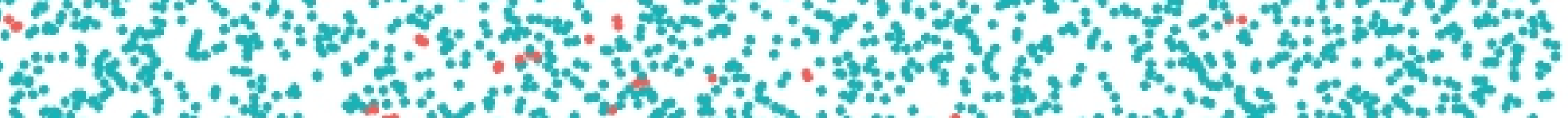

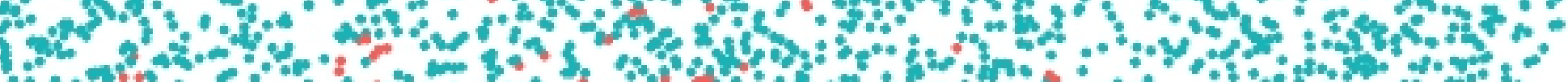
Sho

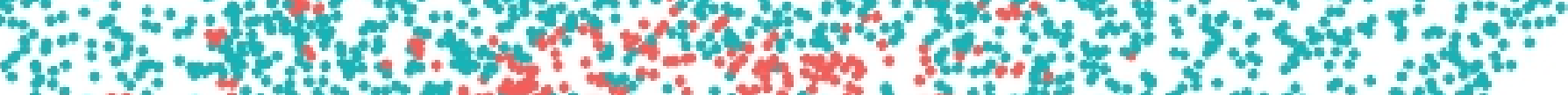

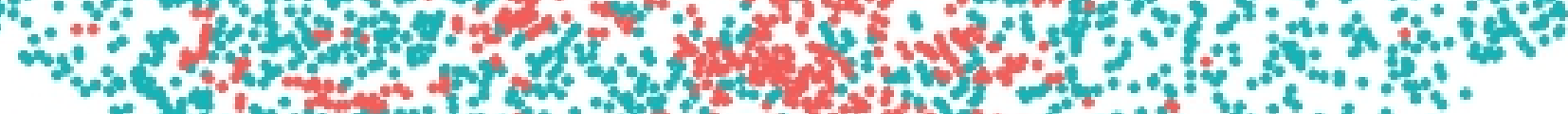

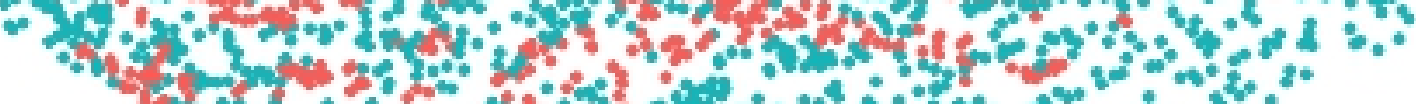

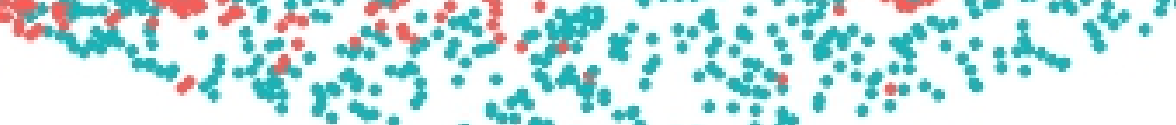




\section{(2)

$$
\text { 2at. }
$$

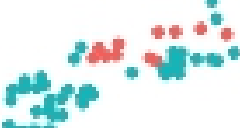

+

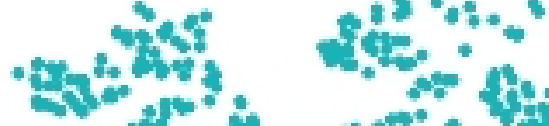
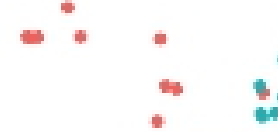

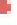
8.

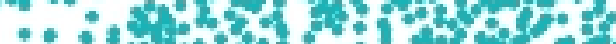

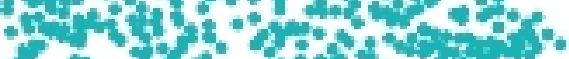

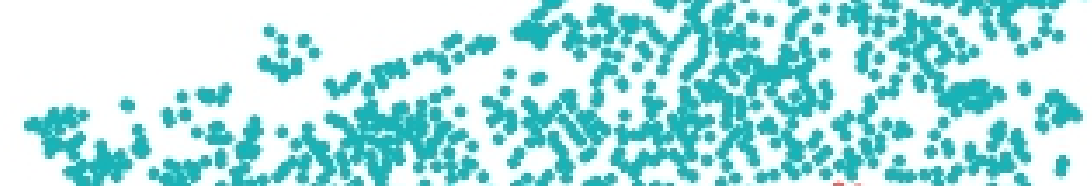

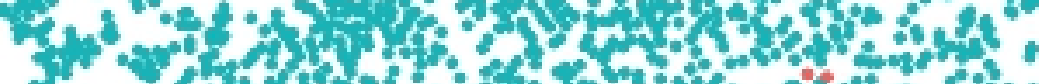

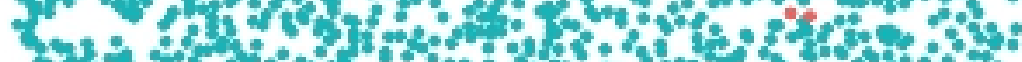
7 f

B. $=1$ (S)

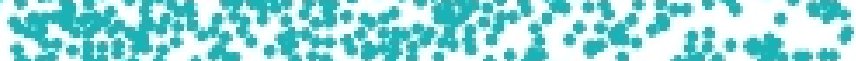

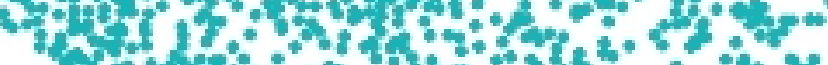
(f) 25:-

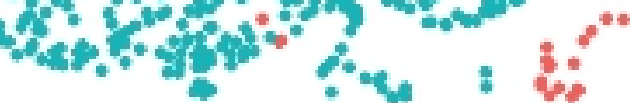

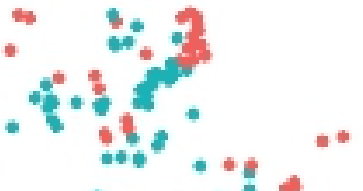

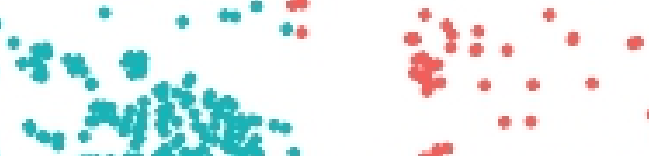

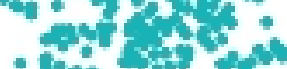

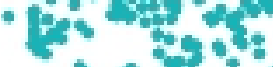
An 4 . 34 .

$$
\text { 指 }
$$

$+2+x^{2}+2$

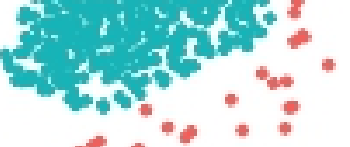

